\title{
Solute-point defect interactions, coupled diffusion, and radiation-induced segregation in fcc nickel
}

\author{
E. Toijer $\odot,{ }^{1}$ L. Messina $\odot,{ }^{2,1}$ C. Domain $\odot,{ }^{3}$ J. Vidal,${ }^{3}$ C. S. Becquart $\odot,{ }^{4}$ and P. Olsson $\oplus^{1}$ \\ ${ }^{1}$ KTH Royal Institute of Technology, Nuclear Engineering, SE-114 21 Stockholm, Sweden \\ ${ }^{2}$ CEA, DES, IRESNE, DEC, Cadarache F-13108 Saint-Paul-Lez-Durance, France \\ ${ }^{3}$ Department of Materials and Mechanics of Components, EDF R\&D, F-77250 Moret sur Loing, France \\ ${ }^{4}$ Université Lille, CNRS, INRAE, Centrale Lille, UMR 8207 - UMET - Unité Matériaux et Transformations, F-59000 Lille, France
}

(Received 4 September 2020; revised 4 November 2020; accepted 15 December 2020; published 19 January 2021)

\begin{abstract}
Radiation-induced segregation (RIS) of solutes in materials exposed to irradiation is a well-known problem. It affects the lifetime of nuclear reactor core components by favoring radiation-induced degradation phenomena such as hardening and embrittlement. In this work, RIS tendencies in face centered cubic (fcc) Ni- $X(X=\mathrm{Cr}$, $\mathrm{Fe}, \mathrm{Ti}, \mathrm{Mn}, \mathrm{Si}, \mathrm{P})$ dilute binary alloys are examined. The goal is to investigate the driving forces and kinetic mechanisms behind the experimentally observed segregation. By means of $a b$ initio calculations, point-defect stabilities and interactions with solutes are determined, together with migration energies and attempt frequencies. Transport and diffusion coefficients are then calculated in a mean-field framework, to get a full picture of solutedefect kinetic coupling in the alloys. Results show that all solutes considered, with the exception of $\mathrm{Cr}$, prefer vacancy-mediated over interstitial-mediated diffusion during both thermal and radiation-induced migration. $\mathrm{Cr}$, on the other hand, preferentially migrates in a mixed-dumbbell configuration. $\mathrm{P}$ and $\mathrm{Si}$ are here shown to be enriched, and $\mathrm{Fe}$ and $\mathrm{Mn}$ to be depleted at sinks during irradiation of the material. Ti and $\mathrm{Cr}$, on the other hand, display a crossover between enrichment at lower temperatures, and depletion in the higher temperature range. Results in this work are compared with previous studies in body centered cubic (bcc) Fe, and discussed in the context of RIS in austenitic alloys.
\end{abstract}

DOI: 10.1103/PhysRevMaterials.5.013602

\section{INTRODUCTION}

Ni-based alloys and austenitic stainless steel are common structural materials in current and future generation nuclear power plants (NPPs). Novel material classes such as highentropy alloys (HEAs), or concentrated solid solution alloys (CSAs) are also materials that can be Ni based with a face centered cubic (fcc) structure. Such materials can in the nuclear technology sector be exposed to intense radiation fields, which can have a great impact on their properties. In Ni-based alloys, experimental observations show radiationinduced creep, swelling, and embrittlement [1,2]. The change in material properties is generally related on the atomic scale to point defect (PD) formation and diffusion [3-5]. The coupling of PDs and atomic fluxes can lead to the redistribution of atoms independently of or alongside thermodynamic forces in a process known as radiation-induced segregation (RIS). This may in turn induce loss of strength and ductility, which consequently can lead to failure during reactor operation. Experimental characterization of irradiated material provides important insight on the processes involved in its degrada-

Published by the American Physical Society under the terms of the Creative Commons Attribution 4.0 International license. Further distribution of this work must maintain attribution to the author(s) and the published article's title, journal citation, and DOI. Funded by Bibsam. tion. However, the observations are in this case very much dependent on the exact conditions during irradiation. In order to better anticipate the evolution of an irradiated material, thermodynamic driving forces and solute-PD coupling must be understood, and atomic-scale modeling is a helpful tool for this task. The approach can provide important information on the interactions of various chemical species in metals, such as $\mathrm{Ni}$-based alloys currently used in NPPs. Atomic-scale investigations of austenitic alloys and HEAs, however, have proven very difficult given their complex chemistry and magnetic properties. A common practice for modeling austenitic steels is to use $\mathrm{Fe}$ as a basis, since it represents the main constituent. However, replicating the fcc structure of austenitic alloys is difficult due to the instability of fcc $\mathrm{Fe}$ at $0 \mathrm{~K}$, where density functional theory (DFT) calculations are generally performed. This can be a problem since results obtained for one structure are not necessarily transferable to another [6]. Additionally, the paramagnetic state of fcc steels at higher temperatures is difficult to represent at $0 \mathrm{~K}$ where the ground state of $\mathrm{Fe}$ is body centered cubic (bcc) and ferromagnetic. Proper modeling of austenitic alloys and HEAs remains for these reasons a challenging task.

In Ni-based, austenitic, and high entropy alloys, a number of different chemical species are present. For this reason, the impact of irradiation on the material cannot be understood only by looking at the behavior of pure Ni. Multiple studies have been undertaken to clarify how common species behave in fcc Ni under irradiation. $\mathrm{Fe}$ and $\mathrm{Cr}$ have been shown to slow down defect migration and decrease the accumulation 
and average size of defect clusters in Ni alloys [7-13]. Si has been shown to suppress void formation and growth, and to enhance grain boundary cohesion [14-21]. P is known to embrittle austenitic alloys [22], but its embrittling effect on $\mathrm{Ni}$ is debated $[20,21,23,24]$. Studies regarding the behavior of $\mathrm{Ti}$ in Ni alloys are scarcer, however, Ti depletion following irradiation has been observed [25], and solute-induced enhancement of theoretical strength has been reported [26]. $\mathrm{Mn}$ is present in all austenitic steels, as well as in Ni-based superalloys, and is also a common ingredient in fcc HEAs. For $\mathrm{Mn}$ in fcc Ni, RIS is indicated to be driven by vacancy exchange in the opposite direction to that of $\mathrm{P}$ [27]. From these previous works, it is clear that $\mathrm{Fe}, \mathrm{Cr}, \mathrm{Si}, \mathrm{P}, \mathrm{Ti}$, and $\mathrm{Mn}$ can strongly impact the microstructural evolution of $\mathrm{Ni}$ alloys. For this reason, a correct description of RIS tendencies of each species is of high importance to anticipate the evolution of the irradiated system.

The solute RIS tendencies can be obtained by computing the full matrix of transport coefficients [28], which describes the kinetic response of each species to thermodynamic driving forces, and the possible presence of coupled fluxes. For fcc alloys, vacancy-solute transport coefficients can be obtained either by means of the self-consistent mean-field (SCMF) method [29,30] or the Green's-function method [31]. However, transport coefficients related to interstitial-assisted diffusion can be obtained only in a SCMF framework [32], which has been recently extended to arbitrarily long interaction ranges and any periodic crystal structure thanks to the KINECLUE code [33]. Based on solute-PD binding and migration energies obtained with DFT, full dumbbell-transport matrices have been computed in bcc alloys [34], but never yet in fcc alloys. The approach extends the SCMF framework established for dumbbell diffusion in bcc and fcc alloys [32], and can be seen as a broad generalization of the traditional five-frequency model [35], which is limited to vacancy diffusion and short-ranged solute-PD interactions. As such, it provides a complete framework by combining vacancy- and interstitial-assisted transport.

The goal of this study is to improve the current understanding of irradiated $\mathrm{Ni}$ alloys by an accurate description of RIS stemming from calculations of transport coefficients in dilute binary alloys. This has been done by applying the SCMF method parametrized with DFT calculations of solutePD binding and migration energies to get a more accurate description of the behavior of $\mathrm{Fe}, \mathrm{Cr}, \mathrm{Si}, \mathrm{Ti}, \mathrm{Mn}$, and $\mathrm{P}$ solutes in fcc Ni. As the ground-state phase of Ni has an fcc structure, and austenitic steels used in today's NPPs feature a high Ni content $(\sim 10 \%)$, results obtained for fcc $\mathrm{Ni}$ are not only of interest for Ni-based alloys. Results here presented will, for this reason, be compared with previous results from bcc $\mathrm{Fe}$ and discussed in the context of radiation damage in austenitic steels.

\section{METHODOLOGY}

Atomic transport properties are examined in the framework of SCMF theory [29] using the KINECLUE code [33]. The code allows for the calculation of transport coefficients, from which flux coupling and RIS tendencies can be determined, based on $a b$ initio solute-defect thermodynamic interactions and migration barriers. In this section, the relevant background and methodology are outlined. Section II A presents the theory of kinetic coupling and diffusion driving forces, Sec. II B gives a short description of how the transport properties were calculated in this work, and in Sec. II C, the ab initio methodology is presented.

\section{A. Kinetic coupling and transport coefficients}

Even though vacancies occur naturally in any metallic material, in a reactor at its operating temperatures $\left(\sim 300^{\circ} \mathrm{C}\right.$ for generation II-III, $\sim 600^{\circ} \mathrm{C}$ for generation IV), the vacancy population is dominated by irradiation-induced effects. In addition, the radiation field is responsible for the generation of an equal amount of self-interstitial atoms (SIAs) that are essentially negligible under thermal conditions. The spatially inhomogeneous formation of defects results in chemical potential gradients (CPGs), which in turn induce atomic and defect fluxes in the material. The flux of a species $\alpha$ due to the CPGs acting on each species $\beta$ is given by Eq. (1):

$$
J_{\alpha}=-\sum_{\beta} L_{\alpha \beta} \frac{\nabla \mu_{\beta}}{k_{B} T},
$$

where $L_{\alpha \beta}$ are the transport coefficients (also known as the coefficients of the Onsager matrix), i.e., the proportionality factors between the flux of a species $\alpha$ and a CPG, $\nabla \mu$, acting on species $\beta$. In a binary alloy, $\alpha=A, B, V$, and $I$, which represent solvent atoms (Ni), solutes, vacancies, and interstitial defects, respectively. In this case, there are three independent coefficients for each defect, namely $L_{V V}, L_{V B}$, $L_{B B}(V)$ for vacancy-assisted diffusion, and $L_{I I}, L_{I B}, L_{B B}(I)$ for interstitial-assisted diffusion. These coefficients describe the kinetic response of a system to thermodynamic forces. The off-diagonal coefficients, $L_{V B}$ and $L_{I B}$, describe the coupling between different species, i.e., when a flux of a given species is induced by the CPG acting on another species. In addition, the knowledge of the full Onsager matrix allows for the calculation of tracer self- and solute diffusion coefficients, as well as for the prediction of solute radiation-induced segregation tendencies.

\section{Radiation-induced segregation}

If the transport coefficients are known, it is possible to model RIS under steady-state conditions in a binary alloy $(A B)$ using Eq. (2) [34,36,37]:

$$
\frac{\nabla c_{B}}{c_{B}}=-\alpha \frac{\nabla c_{V}}{c_{V}},
$$

where $c_{B}$ and $c_{V}$ are the solute and vacancy concentrations, respectively, and

$$
\alpha=\frac{L_{I A} L_{V A}}{\phi\left(L_{I A} D_{B}+L_{I B} D_{A}\right)}\left(\frac{L_{V B}}{L_{V A}}-\frac{L_{I B}}{L_{I A}}\right),
$$

where $D_{A}$ and $D_{B}$ are the intrinsic diffusion coefficients of solvent and solute atoms. These can be computed directly from the transport coefficients (the full set of equations are provided in a previous publication [34]). The thermodynamic factor $\phi$ describing the change of chemical potential of one species with respect to a concentration change of another, can be 
assumed equal to unity in the dilute limit. The model in Eqs. (2) and (3) represents the steady-state solution of Eq. (1), and assumes a low defect-sink density and the absence of relevant sink bias. In addition, accordingly to the dilute limit, multiple-solute and multiple-defect effects are neglected. More details about the derivation and the underlying assumptions of the model can be found in previous publications $[34,36,37]$. From Eq. (2) it can be seen that the sign of $\alpha$ determines if the concentration gradient of species $B$ is in the same direction as that of defects. Based on the assumption that defects diffuse towards sinks, where they are preferentially absorbed, the corresponding concentration gradient is negative, so a positive $\alpha$ indicates enrichment of species $B$ at sinks, whereas a negative $\alpha$ indicates depletion. In Eq. (3), the sign-determining factor of $\alpha$ is given by

$$
\left(\frac{L_{V B}}{L_{V A}}-\frac{L_{I B}}{L_{I A}}\right)
$$

where the left fraction is related to vacancy-coupled fluxes, and the right one to SIA-coupled ones. From the two fractions, it is possible to investigate the segregation tendencies induced by each mechanism independently. The two fractions are termed partial diffusion coefficient (PDC) ratios, and the impact of the respective mechanisms is determined by setting the opposite PDC ratio to 1, describing in this case an uncorrelated flux between the solute and that defect. In the case of vacancy-mediated diffusion, the competition between the solute and the bulk species can result in three distinct cases. In the case of preferential vacancy-solute exchange and positive flux coupling $\left(L_{B V}>0\right)$, solutes migrate in the same direction as vacancies, and solute enrichment at sinks occurs. This process is known as vacancy drag, and is indicated by a negative vacancy PDC ratio since $L_{A V}$ is always negative. When vacancy drag does not take place, enrichment can still take place, as in this case, both solvent and solute atoms diffuse against the vacancy flux (inverse Kirkendall mechanism), thus away from the sink. If the solute is slower than the solvent (preferential solvent-vacancy exchange), the solute will effectively be enriched at sinks. This is indicated by $0<\mathrm{PDC}_{\mathrm{vac}}<1$. In the case, instead, of preferential solute-vacancy exchange, solutes will diffuse away faster than solvent atoms, and depletion occurs $\left(\mathrm{PDC}_{\mathrm{vac}}>1\right)$. For interstitial-mediated diffusion, the flux of solutes cannot be in the opposite direction to that of interstitials, so the $\mathrm{PDC}_{\mathrm{SIA}}$ is always positive. If solute transport is faster than solvent transport, this results in enrichment at sinks, and in this case the $\mathrm{PDC}_{\text {SIA }}$ is greater than 1 . The $\mathrm{PDC}_{\text {SIA }}$ is smaller than 1 in the opposite case.

\section{Self- and solute diffusion coefficients}

Solute and solvent diffusion coefficients in thermodynamic equilibrium have been determined experimentally for many systems. As diffusion coefficients can also be calculated based on the transport coefficients obtained in the context of SCMF theory, they are useful for validation of results. Under thermalequilibrium conditions, migration occurs predominantly by a vacancy-assisted mechanism, and the self- (solvent) and solute diffusion coefficients can be computed with Eqs. (4) and (5) respectively [35]:

$$
D^{*}=g a_{0}^{2} f_{0} \omega_{0} c_{v}^{e q}=g a_{0}^{2} f_{0} \nu_{0} \exp \left(-\frac{E_{v}^{f}+E_{v}^{m}}{k_{B} T}\right) \exp \left(\frac{S_{v}^{f}}{k_{B}}\right),
$$

$$
\begin{aligned}
D_{B}^{*}= & g a_{0}^{2} f_{B} \omega_{2} c_{V}^{e q} p_{1 n n}=g a_{0}^{2} f_{B} \nu_{B} \exp \left(\frac{-E_{V}^{f}-E_{B}^{m}+E_{B V}^{b, 1 n n}}{k_{B} T}\right) \\
& \times \exp \left(\frac{S_{V}^{f}}{k_{B}}\right),
\end{aligned}
$$

where $g$ is a geometrical factor ( $g=1$ for monovacancy diffusion in fcc), $a_{0}$ is the lattice constant, $f_{0}$ and $f_{B}$ are the selfand solute correlation factors, $\omega_{0, B}$ are the jump frequencies of a vacancy exchanging respectively with a solvent atom (in the absence of solutes nearby) or a solute atom, $v_{0, B}$ are the corresponding attempt frequencies, $p_{1, \mathrm{nn}}$ is the probability of having a solute-vacancy pair at a first nearest neighbor (1nn) distance, $E_{V}^{f}$ and $S_{v}^{f}$ are the vacancy formation energy and entropy, $E_{B}^{m}$ is the solute migration barrier, $E_{B V}^{b, 1 n n}$ is the $1 \mathrm{nn}$ solute-vacancy binding energy, here defined as attractive when positive. The solute correlation factor $f_{B}$ is related to the probability for the solute atom to make an immediate reverse jump back to its previous position, thus leading to no net displacement. In the context of the five-frequency model [35], this factor is obtained by considering only the probabilities of the defect returning from second, third, and fourth nearest neighboring positions to the $1 \mathrm{nn}$ position with respect to the solute [35]. In place of Eqs. (4) and (5), the diffusion coefficients can be computed directly from the transport coefficients according to Eqs. (6) and (7) (valid in the case of dilute concentrations), in which $A^{*}$ is the solvent tracer:

$$
\begin{gathered}
D^{*}=\frac{L_{A^{*} A^{*}}}{c_{A^{*}}}, \\
D_{B}^{*}=\frac{L_{B B}}{c_{B}} .
\end{gathered}
$$

The resulting correlation factors $f_{0}$ and $f_{B}$ are included in $L_{A * A *}$ and $L_{B B}$ respectively. Within SCMF theory and its implementation in the KINECLUE code, the calculation of the transport coefficients considers kinetic trajectories of increasing amplitude, up to a cut-off kinetic radius that can be arbitrarily chosen by the user. In addition, whereas in Eq. (5) the probability of a vacancy-solute pair accounts for $1 \mathrm{nn}$ thermodynamic interactions only, KINECLUE allows for a more accurate evaluation of the pair partition function, thus providing a pair probability that takes into account longer-distance vacancy-solute interactions. Thanks to this approach, it is thus possible to provide a more accurate evaluation of the kinetic properties of $\mathrm{Ni}$ alloys with respect to previous works based on the five-frequency model.

\section{B. Calculation of transport properties}

The symmetric Onsager matrix is calculated in this work using the KINECLUE code, which implements SCMF theory to expand the Onsager matrix in terms of cluster 
contributions [38]. The transport coefficients are in this case given by Eq. (8):

$$
L_{i j}=\zeta \sum f_{c} L_{i j}^{(c)},
$$

where $\zeta$ is the total concentration of all monomers and clusters and $f_{c}$ is the concentration fraction of vacancy or solute clusters (which in the dilute limit corresponds to

$$
\left[\begin{array}{ccc}
L_{V V} & L_{V I} & L_{V B} \\
L_{I V} & L_{I I} & L_{I B} \\
L_{V B} & L_{I B} & L_{B B}
\end{array}\right]=\zeta\left(f_{V}\left[\begin{array}{ccc}
L_{V V}^{(V)} & 0 & 0 \\
0 & 0 & 0 \\
0 & 0 & 0
\end{array}\right]+f_{I}\left[\begin{array}{cc}
0 & 0 \\
0 & L_{I I}^{(I)} \\
0 & 0
\end{array}\right.\right.
$$

$\left.\begin{array}{l}0 \\ 0 \\ 0\end{array}\right]+f_{V B}\left[\begin{array}{c}L_{V V}^{(V B)} \\ 0 \\ L_{V B}^{(V B)}\end{array}\right.$

where $f_{X}$ represent the corresponding cluster concentrations. Interstitial-vacancy correlations represented by the $L_{\mathrm{IV}}=L_{\mathrm{VI}}$ coefficients are neglected in this work, under the assumption that the recombination probability is low when the concentrations of the two types of point defects are sufficiently low.

In order to calculate the transport coefficients of Eq. (9), thermodynamic interactions and migration mechanisms must be outlined. In the case of interstitial migration, three possible configurations should be considered in the fcc lattice: octahedral, tetrahedral, and dumbbell. DFT calculations in this work demonstrate that the $\langle 100\rangle$ dumbbell is associated in Ni with the lowest energy of the possible interstitial configurations for all species considered, with the notable exception of P. Indeed, it is shown in Sec. III A that the mixed-P $\langle 100\rangle$ dumbbell displays a severe instability, and that the octahedral configuration is significantly more stable for that species. DFT calculations in this work also demonstrate that if a pure Ni dumbbell comes sufficiently close to a substitutional $\mathrm{P}$, the $\mathrm{P}$ will be kicked out into a octahedral configuration, as one of the $\mathrm{Ni}$ atoms of the dumbbell takes its place in the lattice. For this reason, only dumbbell-induced octahedral diffusion is relevant in the case of $\mathrm{P}$ interstitials. As the $\mathrm{P}$ atoms need the presence of a pure Ni dumbbell to be kicked out into an octahedral configuration, the diffusion process is strongly coupled with pure-Ni dumbbell diffusion. Regarding all other solutes in this work, DFT results indicate that the $\langle 100\rangle$ dumbbell is of main importance for interstitial migration. In this configuration, a

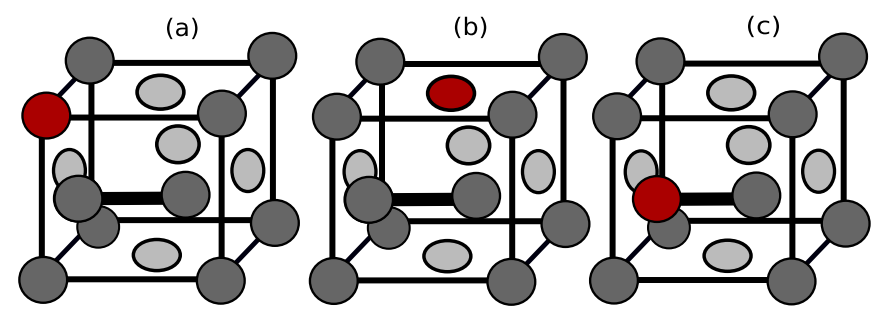

FIG. 1. Three $\langle 100\rangle$ dumbbell configurations in an fcc lattice. (a) A pure nickel dumbbell with the solute in a compressed lattice site ( $a$ type). (b) A pure nickel dumbbell with the solute in a tensed lattice site ( $b$ type). (c) A mixed dumbbell. The red circles indicate the position of the solute atom.

$$
\left.\left.\begin{array}{cc}
0 & L_{V B}^{(V B)} \\
0 & 0 \\
0 & L_{B B}^{(V B)}
\end{array}\right]+f_{I B}\left[\begin{array}{ccc}
0 & 0 & 0 \\
0 & L_{I I}^{(I B)} & L_{I B}^{(I B)} \\
0 & L_{I B}^{(I B)} & L_{B B}^{(I B)}
\end{array}\right]\right),
$$

monovacancies, monointerstitials, vacancy-solute pairs and vacancy-interstitial pairs, respectively). For a full discussion of the breakdown of transport coefficients into cluster contributions, see [34] and [38]. In the dilute limit, the Onsager matrix is split into contributions from isolated vacancies $(V)$, isolated interstitials $(I)$, solute-vacancy pairs $(V B)$, and soluteinterstitial pairs $(I B)$. The matrix is in this case given by Eq. (9) [34]:

solute can be part of the defect as a mixed dumbbell, or in its neighborhood. Concerning $1 \mathrm{nn}$ configurations, which are characterized by the strongest thermodynamic interactions, the solute can be in a compressed ( $a$ type) or tensed ( $b$ type) position depending on the dumbbell orientation in relation to the solute. The dumbbell can for this reason move in multiple ways in relation to the solute. The $\langle 100\rangle$ mixed-dumbbell and $1 \mathrm{nn}$ configurations considered in this work are illustrated in Fig. 1. As it is reasonable to assume that the solute dumbbell interaction quickly drops to zero after the $1 \mathrm{nn}$, as was the case for Fe alloys [34], interactions beyond this distance were not explicitly calculated in this work.

After the thermodynamics of the system has been analyzed, the corresponding migration paths of the energetically favorable configurations are determined in order to calculate the transport coefficients. The set of symmetry-unique configurations and jump frequencies needed for the calculation of the transport coefficients can be determined either explicitly using ab initio (DFT), or by the kinetically resolved activation (KRA) approach [39] implemented in the KINECLUE code. The jumps which are explicitly calculated with DFT in this work are illustrated in Fig. 2 in the case of solute-vacancy related migration, and Fig. 3 in the case of solute $\langle 100\rangle$ dumbbell related migration. KINECLUE evaluates all other possible jumps

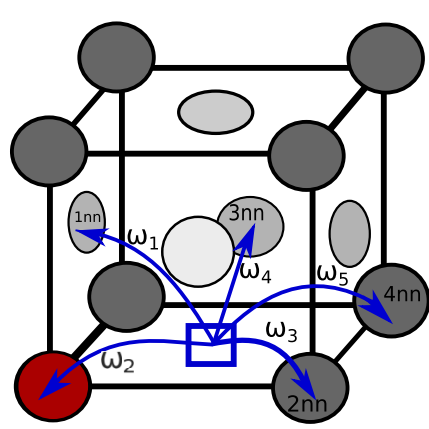

FIG. 2. Illustration of the solute-vacancy related migration barriers, denoted by their respective jump frequencies $\omega$, explicitly calculated using DFT. The red circle indicates the position of the solute atom, the blue square that of the vacancy. The neighbor ordering is with respect to the solute atom. 


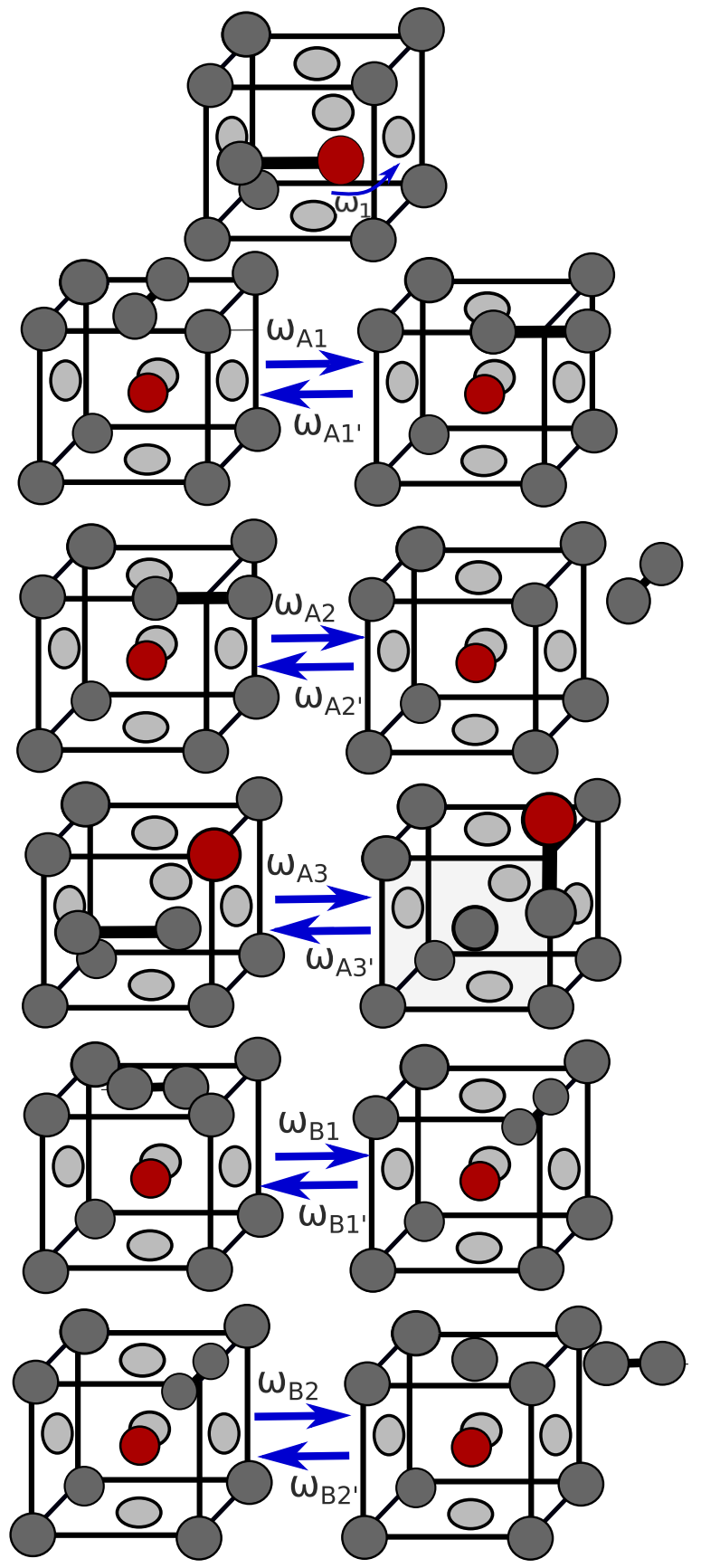

FIG. 3. Illustration of the solute dumbbell related migration barriers explicitly calculated using DFT. The red circle indicates the position of the solute atom.

up to a maximum trajectory range, i.e., the kinetic radius, via the KRA approach. As the transport coefficients have been shown to be well converged within a kinetic radius of $4 a_{0}$ $[33,34]$, this value was used in all calculations.

Once the thermodynamic interactions and migration mechanisms have been evaluated, the cluster transport coefficients of Eq. (9) can be calculated with KINECLUE, following the process outlined in [34]. Calculations were performed in the dilute limit, with a solute-to-solvent ratio of $10^{-4}$. As the transport coefficients are given as output from KINECLUE calculations, RIS tendencies of the system are thereafter evaluated following the approach of Sec. II A.

\section{Ab initio methodology}

The calculation of transport coefficients relies on thermodynamic parameters (formation and binding energies) and kinetic parameters (jump frequencies).

For a configuration containing $n$ atoms of the solvent $X$ and $p$ sites of the solute $Y$, the formation energy is given by Eq. (10).

$$
E_{f}(n X+p Y)=E[n X+p Y]-n E[X]-p E_{\mathrm{imp}}[Y],
$$

where $E_{\text {imp }}$ is the reference state of the solute. The crystal structures of the used reference states are body centered cubic (bcc) for $\mathrm{Cr}, \mathrm{Mn}$, and $\mathrm{Fe}$, tetrahedral structure ( $p 4)$ for $\mathrm{P}$, diamond structure for $\mathrm{Si}$, and hexagonal closed packed (hcp) structure for Ti.

The binding energy between $n$ objects in a supercell is given in Eq. 11.

$$
\begin{aligned}
E_{b}\left(A_{1}, A_{2}, \cdots, A_{n}\right)= & \sum_{i=1, \cdots, n} E\left(A_{i}\right) \\
& -\left[E_{\mathrm{ref}}+E\left(A_{1}+A_{2}+\cdots+A_{n}\right)\right],
\end{aligned}
$$

where $E_{\text {ref }}$ is the energy of the supercell without any defects, $E\left(A_{i}\right)$ is the energy of the supercell with the isolated defect $A_{i}$, and $E\left(A_{1}+A_{2}+\cdots+A_{n}\right)$ is the energy of the cell containing all $A_{i}$ interacting defects. With this definition, positive values are binding (or attractive) configurations.

The jump frequency is defined by Eq. (12):

$$
\omega=v \exp \left(-E_{\mathrm{mig}} / k_{B} T\right),
$$

where $E_{\text {mig }}$ is the migration barrier and $v$ is the attempt frequency, given by Eq. (13):

$$
v=\frac{\prod v_{j}^{R}}{\prod v_{k}^{S}},
$$

where, $v_{j}^{R}$ are the vibrational eigenfrequencies in the relaxed defect supercell, and $v_{j}^{S}$ are the eigenfrequencies in the saddlepoint configuration.

The above properties (formation, binding and migration energies, and attempt frequencies) were obtained through $a b$ initio density functional theory (DFT) calculations using the Vienna $A b$ initio Simulation Package (VASP) [40,41], with pseudopotentials from the VASP library generated with the projector augmented wave (PAW) method using the PerdewBurke-Ernzerhof (PBE) exchange-correlation functional [42]. Spin polarization, periodic boundary conditions, and the supercell approach were applied for all calculations. The Brillouin zone was sampled with $3 \times 3 \times 3 k$ points using the Monkhorst-Pack scheme. Supercells of $256(4 \times 4 \times 4) \mathrm{fcc}$ lattice sites were used for the calculations, unless otherwise stated. The plane-wave energies were cut off at $350 \mathrm{eV}$, and all relaxations were performed under constant volume conditions with a Ni lattice parameter of $3.522 \AA$. Calculations were performed in the ferromagnetic state; however, as magnetic moments of $\mathrm{Cr}$ solutes can be sensitive to the initial state, an initial guess of $-2 \mu_{\mathrm{B}}$ was used for this species to obtain the 
TABLE I. Point-defect and impurity formation energies $(\mathrm{eV})$ in Ni. Unstable configurations are omitted and marked with “-”. See Fig. 1 for an illustration of the three $\langle 100\rangle$ dumbbell configurations. All calculations were performed in a 108-atom cell and with an energy cutoff of $350 \mathrm{eV}$.

\begin{tabular}{lcccccc}
\hline \hline Defect & $\mathrm{Ni}$ & $\mathrm{Cr}$ & $\mathrm{Fe}$ & $\mathrm{P}$ & $\mathrm{Si}$ & $\mathrm{Ti}$ \\
\hline$\langle 100\rangle$ & 4.2 & 3.9 & 3.8 & - & 1.8 & 3.0 \\
$\langle 110\rangle$ & 5.0 & 4.6 & 4.8 & 1.1 & 2.6 & 3.4 \\
$\langle 11\rangle\rangle$ & 4.8 & 4.2 & 4.4 & 1.4 & 2.6 & - \\
Octahedral & 4.4 & 4.1 & 4.0 & 0.2 & 1.9 & 3.0 \\
Tetrahedral & 4.8 & 4.2 & 4.3 & 1.5 & 2.9 & 3.0 \\
Substitutional & - & 0.4 & -0.4 & -1.8 & -1.6 & -1.4 \\
Vacancy & 1.4 & - & - & - & -0.5 \\
A-type $\langle 100\rangle$ (compressed) & - & 4.2 & 3.8 & - & 2.4 & - \\
$B$-type $\langle 100\rangle$ (tensed) & - & 4.3 & 3.8 & 2.9 & 3.0 & 2.8 \\
\hline \hline
\end{tabular}

lower energy state. In order to calculate migration barriers, the nudged elastic band method (NEB) $[43,44]$ implemented in VASP was used with three images and the climbing-image algorithm [45] to obtain the saddle-point energy. This was checked to be sufficient for a precision of $20 \mathrm{meV} / \AA$ in force convergence. Attempt frequencies for vacancy migration were determined by the finite-difference method using the PHONOPY software [46] in $256( \pm 1)$ atom supercells with a displacement per atom of $\pm 0.01 \AA$. The initial structure and the structure at the saddle point were relaxed with a high accuracy in terms of residual forces $\left(10^{-7} \mathrm{eV}\right.$ on the total energy, and each force below $0.3 \mathrm{meV} / \AA$ ).

\section{RESULTS}

\section{A. Point defect equilibrium properties}

The defects considered in this work are the mixed and pure $\langle 100\rangle,\langle 110\rangle$, and $\langle 111\rangle$ dumbbells, the octahedral, tetrahedral, and substitutional impurities, as well as the vacancy in pure $\mathrm{Ni}$. The defect formation energies in bulk $\mathrm{Ni}$ are presented in Table I, and the vacancy-solute and self-interstitial atom (SIA) solute binding energies are presented in Table II. Vacancy solute binding energies are presented as functions of the mutual distance between the two. Solute interactions with the pure $\langle 100\rangle \mathrm{Ni}$ dumbbell were also considered with a solute atom as a first nearest neighbor (1nn) in compressed and tensed lattice sites. For an illustration of the considered solute $\langle 100\rangle$ dumbbell configurations, see Fig. 1.

In this work the vacancy formation energy in $\mathrm{Ni}$ was calculated to $1.4 \mathrm{eV}$. This value is in line with calculations by Nazarov et al. where the PBE exchange correlation functional was also used [47]. Results in Table I show that the preferred SIA configuration in pure $\mathrm{Ni}$ is the $\langle 100\rangle$ dumbbell. This is in contrast to bcc Fe, where the $\langle 110\rangle$ dumbbell is the most stable $[34,48,49]$. The introduction of $\mathrm{Cr}$ and $\mathrm{Si}$ solute atoms in $\mathrm{Ni}$ does not change the relative stability of the $\langle 100\rangle$ dumbbell. From the strong stability of the mixed $\langle 100\rangle$ dumbbells for the two species, one can suspect an efficient solute transport due to this migration mechanism. The strong interaction of the mixed $\mathrm{Si}-\mathrm{Ni}$ dumbbell is different from observations of $\mathrm{Si}$ in bcc $\mathrm{Fe}$, where the $\mathrm{Fe}-\mathrm{Si}$ dumbbell is neither binding nor repulsive [34]. For $\mathrm{Cr}$, on the other hand, the formation of a stable $\langle 100\rangle$ dumbbell complex is in line with observations in bcc Fe, where the species has been shown to form a stable mixed $\langle 110\rangle$ dumbbell [34]. Additionally, $\mathrm{Cr}$ has been shown to form a stable mixed $\langle 100\rangle$ dumbbell

TABLE II. Solute-defect binding energies $(\mathrm{eV})$ in pure Ni; positive values represent binding configurations. Unstable configurations are omitted and marked with "-". See Fig. 1 for an illustration of the three $\langle 100\rangle$ dumbbell configurations. The binding energy for vacancy-solute binding is given as a function of nearest neighbor (nn) distance between the two. In the case of the octahedral and tetrahedral configurations, the $\langle 100\rangle$ pure dumbbell and the substitutional solutes were used as reference.

\begin{tabular}{|c|c|c|c|c|c|c|}
\hline & $\mathrm{Cr}$ & $\mathrm{Fe}$ & $\mathrm{P}$ & $\mathrm{Si}$ & $\mathrm{Ti}$ & $\mathrm{Mn}$ \\
\hline $1 \mathrm{nn}$ & -0.04 & -0.02 & 0.28 & 0.10 & 0.05 & -0.01 \\
\hline $2 \mathrm{nn}$ & 0.02 & -0.01 & 0.03 & 0.01 & -0.06 & -0.03 \\
\hline $3 \mathrm{nn}$ & -0.03 & -0.03 & -0.03 & -0.03 & -0.03 & -0.02 \\
\hline $4 \mathrm{nn}$ & 0.02 & 0.03 & 0.01 & 0.01 & 0.07 & 0.03 \\
\hline $5 \mathrm{nn}$ & 0.00 & -0.01 & -0.01 & -0.01 & -0.01 & -0.01 \\
\hline $6 \mathrm{nn}$ & 0.00 & 0.00 & 0.00 & 0.00 & 0.00 & 0.00 \\
\hline Mixed $\langle 100\rangle$ & 0.38 & -0.07 & - & 0.78 & -0.33 & -0.30 \\
\hline Mixed $\langle 110\rangle$ & -0.30 & -0.80 & 1.16 & -0.04 & -0.72 & -0.98 \\
\hline Mixed $\langle 111\rangle$ & 0.14 & -0.60 & 0.96 & 0.01 & - & - \\
\hline$A$-type $\langle 100\rangle$ (compressed) & 0.10 & -0.02 & - & 0.40 & -0.07 & 0.02 \\
\hline$B$-type $\langle 100\rangle$ (tensed) & 0.00 & 0.02 & -0.50 & -0.36 & 0.02 & 0.16 \\
\hline Octahedral & 0.18 & -0.29 & 2.11 & 0.69 & -0.34 & -0.51 \\
\hline Tetrahedral & 0.14 & -0.52 & 0.88 & -0.24 & -0.26 & -0.63 \\
\hline
\end{tabular}




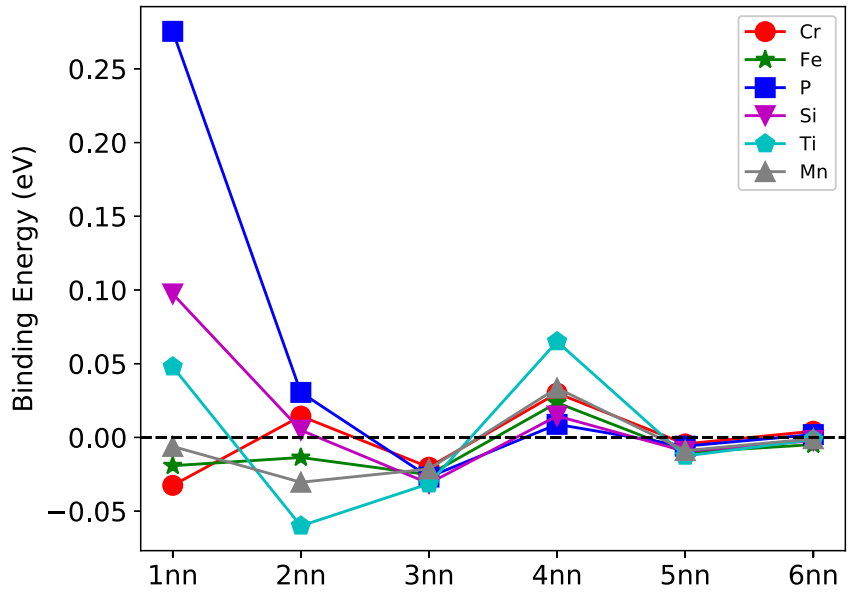

FIG. 4. Vacancy-solute binding energies $(\mathrm{eV})$ in pure $\mathrm{Ni}$ as a function of nearest neighbor (nn) distance. Positive values represent binding configurations.

in fcc $\mathrm{Fe}$ (antiferromagnetic matrix) [50] and in fcc $\mathrm{FeNiCr}$ (special quasirandom structure) [51]. This indicates that the interstitial migration of $\mathrm{Cr}$ may show a similar character in $\mathrm{Fe}$ and $\mathrm{Ni}$. On the other hand, the introduction of $\mathrm{Fe}, \mathrm{P}, \mathrm{Ti}$, and Mn impacts the relative stability of the $\langle 100\rangle$ dumbbell. As shown in Table II, Fe, Ti, and Mn display repulsive interstitial interactions in all but the $B$-type $1 \mathrm{nn}$ configuration, where the binding is anyway very weak. Note that these results apply to $0-\mathrm{K}$ ground-state properties only, and relative stabilities may be altered by finite-temperature effects. In the case of $\mathrm{Ti}$ and $\mathrm{Mn}$, however, the mixed $\langle 100\rangle$ dumbbells are particularly repulsive. As a consequence, the dissociation of the mixed dumbbell is far more likely than its migration, and a net solute displacement induced by the interstitial mechanism is unlikely. For this reason, interstitial migration of Ti and $\mathrm{Mn}$ will not be considered in this work. The result is in contrast to what has been observed in bcc Fe, where $\mathrm{Mn}$ has been shown to form stable mixed dumbbells and migrate efficiently via the dumbbell mechanism [34]. Regarding P, it can be seen in Tables I and II that the species displays the highest stability and strongest binding as an octahedral compared to any other interstitial configuration. This indicates that the mixed-dumbbell mechanism is likely not dominant in the migration of $\mathrm{P}$ atoms. Indeed, DFT calculations in this work evidenced that if the pure Ni dumbbell comes sufficiently close to a $\mathrm{P}$ atom in a substitutional site, the $\mathrm{P}$ will be subjected to a "kickout" mechanism, where the P ends up in an octahedral configuration, while being replaced in the original substitutional site by one of the $\mathrm{Ni}$ atoms of the dumbbell. As a consequence, the main interstitial migration path of $P$ will be through octahedral sites, once the kickout has taken place. In bcc $\mathrm{Fe}, \mathrm{P}$ has been proven most stable in a mixed $\langle 110\rangle$ dumbbell configuration [34,52,53]. Although migration of the $\langle 110\rangle$ mixed dumbbell in Fe takes place in competition with octahedral migration [54], the lack of stability of the mixed Ni-P dumbbell evidenced in this work indicates a very different behavior of $\mathrm{P}$ in the two materials.

The vacancy-solute binding energies from Table II are illustrated in Fig. 4. It can be seen that P displays the strongest binding, followed by $\mathrm{Si}$ and $\mathrm{Ti}$. It should however be noted


FIG. 5. Ni self-diffusion coefficient $\left(D^{*}\right)$ due to thermal vacancies as a function of temperature, compared with experimental data from [63-68]. The parameters used for each curve correspond to those presented in Table V. The lower inset shows the vacancy concentration necessary to fit the calculated self-diffusion coefficient to the experimental values in the figure, when using the $0-\mathrm{K}$ vacancy migration rate $\omega_{0}$ computed in this work.

that the interaction ranges for all vacancy-solute pairs lie in a very short span. A notable exception is the Ti-vacancy interaction, which is attractive in the $1 \mathrm{nn}$ configuration, but displays a stronger repulsion at $2 \mathrm{nn}$ distance. This type of binding-repulsion combination has been previously observed for early transition metals, including $\mathrm{Ti}$, in bcc Fe [55]. In Fe, the effect was attributed to an electronic origin, as opposed to the more common strain-release effects that result from size differences between the elements. Since Ti displays this type of behavior in both $\mathrm{Fe}$ and $\mathrm{Ni}$, one may suspect a similar trend for all early transition metals in fcc $\mathrm{Ni}$, as the systematic study by Wu et al. [56] seems to confirm (see Fig. 5 therein). Figure 4 further displays a slight spike in binding for the $4 \mathrm{nn}$ interaction compared to the $3 \mathrm{nn}$ and $5 \mathrm{nn}$ ones. The effect, which is particularly noticeable for Ti, results from the fact that at $4 \mathrm{nn}$ distance, the solute and vacancy lie along the closed packed $\langle 110\rangle$ direction. The defects are in this case separated by a $\mathrm{Ni}$ atom, and the results in the current work indicate that an attractive interaction can be elastically mediated by the intermediate solvent atom, similarly to what has previously been observed for the corresponding $5 \mathrm{nn}$ configuration for several solutes in bcc Fe [57,58]. However, since the $4 \mathrm{nn}$ interaction has a negligible effect on the overall vacancy-solute coupling and diffusion, a more thorough evaluation of this effect is beyond the scope of the current work. In bcc $\mathrm{Fe}, \mathrm{Mn}, \mathrm{P}, \mathrm{Si}, \mathrm{Cr}$, and $\mathrm{Ni}$ have been shown to form stable vacancy-solute pairs [34,52]. The weak repulsion between the vacancy and $\mathrm{Cr}$ is however in agreement with results from DFT calculations in fcc $\mathrm{Fe}$ [50] and fcc FeNiCr [51]. Overall, the solute-defect 
TABLE III. Vacancy migration energies $(\mathrm{eV})$ for the respective jump frequencies $\omega$ in the vicinity of various solutes in $\mathrm{Ni}$ and solutejump attempt frequencies $v$ (THz). See Fig. 2 for an illustration of the jumps and description of notations. Calculations marked with "**” were performed in a 108-atom cell, all others were performed in 256atom cell. An energy cutoff of $350 \mathrm{eV}$ was used in all calculations.

\begin{tabular}{lrrrrrrr}
\hline \hline & $\mathrm{Ni}$ & $\mathrm{Cr}$ & $\mathrm{Fe}$ & $\mathrm{P}$ & $\mathrm{Si}$ & $\mathrm{Ti}$ & $\mathrm{Mn}$ \\
\hline$v_{0}\left(\right.$ vacancy jump $\left.\omega_{0}\right)$ & 14.32 & & & & & & \\
$\omega_{0}$ (Ni self-migration) & 1.05 & & & & & & \\
$v_{0}$ (solute jump $\left.\omega_{2}\right)$ & & 10.85 & 12.45 & 0.67 & 2.64 & 7.07 & $6.77^{*}$ \\
$\omega_{2}$ (solute jump) & & 0.77 & 0.93 & 0.58 & 0.79 & 0.5 & 0.75 \\
$\omega_{1}(1 \mathrm{nn} \rightarrow 1 \mathrm{nn})$ & 1.04 & 1.09 & 0.70 & 0.91 & 1.26 & 1.19 \\
$\omega_{3}(1 \mathrm{nn} \rightarrow 2 \mathrm{nn})$ & 1.09 & 1.05 & 1.28 & 1.17 & 1.02 & 1.02 \\
$\omega_{3}^{\prime}(2 \mathrm{nn} \rightarrow 1 \mathrm{nn})$ & 1.14 & 1.05 & 1.03 & 1.07 & 0.91 & 1.00 \\
$\omega_{4}(1 \mathrm{nn} \rightarrow 3 \mathrm{nn})$ & 1.12 & 1.03 & 1.13 & 1.10 & 1.00 & 1.02 \\
$\omega_{4}^{\prime}(3 \mathrm{nn} \rightarrow 1 \mathrm{nn})$ & & 1.13 & 1.03 & 0.83 & 0.98 & 0.92 & 1.01 \\
$\omega_{5}(1 \mathrm{nn} \rightarrow 4 \mathrm{nn})$ & & 1.11 & 1.03 & 1.11 & 1.08 & 0.98 & 1.01 \\
$\omega_{5}^{\prime}(4 \mathrm{nn} \rightarrow 1 \mathrm{nn})$ & & 1.17 & 1.07 & 0.84 & 1.00 & 1.00 & 1.05 \\
\hline \hline
\end{tabular}

interactions in fcc $\mathrm{Ni}$ are different from those in bcc Fe, with the exception of the $\mathrm{P} / \mathrm{Si}$-vacancy interactions, the mixed $\mathrm{Cr}$ $\mathrm{Ni} / \mathrm{Fe}$-dumbbells, and the Ni-Fe dumbbells, which are stable in both materials.

\section{B. Point defect migration kinetics}

The migration barriers for vacancy-mediated diffusion of solutes in fcc $\mathrm{Ni}$ are presented in Table III. Results in Table III show that the attempt frequency for the vacancy-Fe jump is similar to that of pure $\mathrm{Ni}$; it is decreasingly lower for $\mathrm{Cr}$, followed by $\mathrm{Ti}, \mathrm{Mn}$, and $\mathrm{Si}$. $\mathrm{P}$ has a considerably lower attempt frequency compared to the other solutes. The observations are in line with the Meyer-Nelder rule, according to which a high attempt frequency tends to compensate for higher barriers [59]. Ti and $\mathrm{Si}$ however, seem to contradict this rule. The trends observed in Table III, with the largest magnitude of the Ni self-migration barrier, followed by Fe and $\mathrm{Cr}$ in a decreasing order, are in line with trends in fcc Fe [50]. Table III also shows that for all species, with the exception of $\mathrm{Fe}$, the $\omega_{2}$ jumps have considerably lower barriers compared to the corresponding $\omega_{1,3-5}$ barriers. This can be an indication that the species are susceptible to vacancy drag. However, a significantly lower $\omega_{2}$ barrier can result in a continuous solutevacancy exchange, with vacancy trapping causing negligible solute net displacement. For this reason, it is not possible to assess flux coupling and segregation tendencies of these solutes based solely on the results in Tables I-III. In the case of solute $\mathrm{Fe}$, on the other hand, all barriers have similar magnitude, which indicates that the vacancy is less affected by the presence of the species. As it is also shown in Table II that the Fe vacancy interaction is particularly weak, Fe migration by vacancy drag can be considered less likely compared to other species.

The migration barriers and attempt frequencies of the species considered for interstitial-mediated diffusion are presented in Table IV. A number of migration barriers for Ti and Mn were also included for the sake of consistency.

The migration barrier of $0.95 \mathrm{eV}$ for $\mathrm{P}$ octahedral migration is considerably higher than all other barriers in Table IV. This barrier is also significantly higher than that of $\mathrm{P}$ octahedra migration in bcc Fe, which is $0.16 \mathrm{eV}$ [54]. One may for this reason not only suspect a far less efficient diffusion of interstitial $\mathrm{P}$ in fcc Ni compared to bcc Fe, but also of interstitial $\mathrm{P}$ compared to interstitial migration of the other solutes considered in this study-with interstitial Fe being a possible exception. As shown in Table IV, all migration barriers of $\mathrm{Fe}$ have similar magnitude, and the values are close to the barrier of the pure-Ni dumbbell jump. Fe is for this reason unlikely to be susceptible to an efficient dumbbell migration. Regarding $\mathrm{Cr}$, the lowest barrier by far is the

TABLE IV. Self- (Ni) and interstitial migration energies (eV) for the respective jump frequencies $\omega$ and attempt frequencies $v(\mathrm{THz})$ for solutes in Ni obtained in this work. The values marked with " $\dagger$ " refer to octahedral jumps, whereas all others regard the dumbbell mechanism. See Fig. 3 for an illustration of the dumbbell jumps and description of notations. All calculations were performed in a 256-atom cell and with an energy cutoff of $350 \mathrm{eV}$. A migration barrier of $0.0 \mathrm{eV}$ indicates an spontaneous jump without any thermally activated barrier. Barriers marked with "_" were not calculated in this work.

\begin{tabular}{|c|c|c|c|c|c|c|c|}
\hline & $\mathrm{Ni}$ & $\mathrm{Cr}$ & $\mathrm{Fe}$ & $\mathrm{P}$ & $\mathrm{Si}$ & $\mathrm{Ti}$ & $\mathrm{Mn}$ \\
\hline$v_{0}\left(\right.$ for SIA jump $\left.\omega_{0}\right)$ & 2.04 & & & & & & \\
\hline$\omega_{0}$ (pure Ni jump) & 0.14 & & & & & & \\
\hline$\omega_{0 \mathrm{R}}($ pure Ni rot) & 0.61 & & & & & & \\
\hline$v_{1}\left(\right.$ for solute jump $\left.\omega_{1}\right)$ & & 1.77 & 1.42 & $2.21^{\dagger}$ & 0.91 & - & - \\
\hline$\omega_{1}$ (mixed jump) & & 0.08 & 0.20 & - & 0.03 & 0.004 & 0.13 \\
\hline$\omega_{\text {octa }}($ octahedral $\rightarrow$ octahedral $)$ & & - & - & $0.95^{\dagger}$ & - & - & - \\
\hline$\omega_{\mathrm{R}}($ mixed rot $)$ & & 0.24 & 0.53 & - & 0.77 & - & - \\
\hline$\omega_{\mathrm{A} 1}(1 \mathrm{nnA} \rightarrow 1 \mathrm{nnA})$ & & 0.09 & 0.17 & - & 0.02 & 0.24 & - \\
\hline$\omega_{\mathrm{A} 2}(1 \mathrm{nnA} \rightarrow \mathrm{inf})$ & & 0.30 & 0.14 & - & 0.39 & 0.11 & 0.10 \\
\hline$\omega_{\mathrm{A} 2}^{\prime}(\mathrm{inf} \rightarrow 1 \mathrm{nnA})$ & & 0.14 & 0.17 & - & 0.02 & 0.22 & 0.20 \\
\hline$\omega_{\mathrm{A} 3}(1 \mathrm{nnA} \rightarrow$ mixed $)$ & & 0.003 & 0.20 & - & 0.04 & 0.33 & 0.12 \\
\hline$\omega_{\mathrm{A} 3}{ }^{\prime}(\operatorname{mixed} \rightarrow 1 \mathrm{nnA})$ & & 0.29 & 0.15 & - & 0.42 & 0.0 & 0.34 \\
\hline$\omega_{\mathrm{B} 1}(1 \mathrm{nn} \mathrm{B} \rightarrow 1 \mathrm{nnB})$ & & 0.17 & 0.15 & - & 0.17 & 0.17 & 0.16 \\
\hline$\omega_{\mathrm{B} 2}(1 \mathrm{nnB} \rightarrow \mathrm{inf})$ & & 0.23 & 0.18 & - & 0.04 & 0.26 & 0.25 \\
\hline$\omega_{\mathrm{B} 2}{ }^{\prime}(\mathrm{inf} \rightarrow 1 \mathrm{nnB})$ & & 0.11 & 0.14 & - & 0.36 & 0.03 & 0.08 \\
\hline
\end{tabular}


TABLE V. Values used to calculate the temperature dependence of the Ni self-diffusion coefficients of Fig. 5.

\begin{tabular}{|c|c|c|c|c|c|}
\hline & & This work & This work $+C_{V}^{\mathrm{Gong}}$ & Tucker $^{\mathrm{a}}$ & Fitting of expt. data \\
\hline Vacancy formation energy & $E_{V}^{f}$ & $1.40 \mathrm{eV}$ & varying with $T^{\mathrm{a}}$ & $1.65 \mathrm{eV}^{\mathrm{a}}$ & - \\
\hline Vacancy formation entropy & $S_{V}^{f}$ & $1.83 k_{\mathrm{B}}^{\mathrm{a}}$ & varying with $T^{b}$ & $1.83 k_{\mathrm{B}}^{\mathrm{a}}$ & - \\
\hline Vacancy migration energy $(\mathrm{eV})$ & $E_{V}^{\mathrm{mig}}$ & 1.05 & 1.05 & $1.09^{\mathrm{a}}$ & - \\
\hline Attempt frequency $(\mathrm{THz})$ & $v_{0}$ & 14.32 & 14.32 & $4.48 \mathrm{THz}^{\mathrm{a}}$ & - \\
\hline Electronic excitation contribution $\left(\mathrm{eV}^{-1}\right)$ & $E_{c}$ & $0.66^{\mathrm{a}}$ & $0.66^{\mathrm{a}}$ & $0.66^{\mathrm{a}}$ & - \\
\hline Activation energy (eV) (Fig. 5) & $Q$ & 2.44 & 2.49 & 2.74 & 2.83 \\
\hline Prefactor $\left(\mathrm{m}^{2} / \mathrm{s}\right)$ (Fig. 5) & $D_{0}$ & $7.3 \times 10^{-6}$ & $7.7 \times 10^{-6}$ & $2.3 \times 10^{-6}$ & $5.6 \times 10^{-5}$ \\
\hline
\end{tabular}

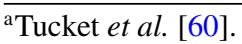

${ }^{\mathrm{b}}$ Gong et al. [61].

association jump $\omega_{\mathrm{A} 3}$ followed by the mixed-dumbbell jump $\omega_{1}$, both of which are lower compared to the pure Ni dumbbell jump $\omega_{0}$. In general, the results in Table IV show that the various association jumps of $\mathrm{Cr}$ have lower barriers than the respective dissociation jumps. A similar trend is seen for $\mathrm{Si}$, where the only barrier lower than the association jump $\omega_{\mathrm{A} 3}$ is the mixed-dumbbell jump $\omega_{1}$. Also for this species, the two barriers are considerably lower than those of all other jumps, with the exception of the $\omega_{\mathrm{B} 2}$ jump. The fact that the $\mathrm{Si}$ dissociation barrier is lower for the $\omega_{\mathrm{B} 2}$ jump can be explained by the repulsive interaction of the species in the $1 \mathrm{nn} B$ configuration, as shown in Table II. In Table II it can also be seen that both $\mathrm{Cr}$ and $\mathrm{Si}$ display strong binding to the $\langle 100\rangle$ dumbbell. The low association barriers, together with the stability of the mixed $\langle 100\rangle$ complexes, are indications of significant dumbbell transport of the two species. However, similarly to the previous discussion for vacancies, a strong correlation can prevent net displacement. Nevertheless, as the mixed dumbbell migrates via rotation translation, the change of orientation makes correlation effects weaker as the probability for zero-displacement back jumps is lower compared to that of vacancy migration [34]. Correlation effects are, for this reason, expected to be less important for dumbbell- compared to vacancy-mediated diffusion.

\section{Solute-transport properties}

From the DFT data presented in Secs. III A and III B, the transport coefficients of each binary alloy were calculated. In Sec. III C 1, the accuracy of the calculated transport coefficients is assessed based on results from experiments. In Sec. III C 2, the dominant diffusion mechanisms of the various species during thermal equilibrium are discussed, and in Sec. III C 3, radiation-induced segregation tendencies are evaluated.

\section{Validation with diffusion experiments}

As diffusion coefficients can be determined experimentally, these can be used to validate the results of this study. However, it should be noted that experiments are mostly performed in the high-temperature range, whereas the diffusion and transport coefficients computed in this work are based on $0-K$ DFT parameters. By relying on the $0-K$ data only, the self-diffusion coefficient, Eq. (4), can be written as

$$
D^{*}=g a_{0}^{2} f_{0} \omega_{0} c_{V}^{\mathrm{eq}},
$$

where the equilibrium vacancy concentration $c_{V}^{\mathrm{eq}}$ and the vacancy migration rate in pure $\mathrm{Ni}, \omega_{0}$, are given by Eqs. (15) and (16), where relevant quantities are defined in Table V:

$$
\begin{aligned}
& c_{V}^{\mathrm{eq}}=\exp \left(-E_{V}^{f} / k_{B} T+S_{V}^{f} / k_{B}\right), \\
& \omega_{0}=v_{0} \exp \left(-E_{V}^{\mathrm{mig}} / k_{B} T\right) E C_{\text {corr }},
\end{aligned}
$$

where $E C_{\text {corr }}$ is a correction term which transposes $a b$ initio $0-\mathrm{K}$ results to finite temperatures by taking into account the contribution of electronic excitations. The factor is given by Eq. (17):

$$
E C_{\text {corr }}=\exp \left(\pi^{2} k_{B} T E_{c} / 6\right),
$$

where $E_{c}$ is the difference between the electronic density of states at the saddle point and the equilibrium position in the lattice site [60].

From the $0-\mathrm{K}$ results of this work $\left(E_{V}^{f}=1.4 \mathrm{eV}, v_{0}=\right.$ $14.32 \mathrm{THz}$, and $E_{V}^{\mathrm{mig}}=1.05 \mathrm{eV}$ ), together with data from Tucker et al. $\left(S_{V}^{f}=1.82 k_{\mathrm{B}}, E_{c}=-0.66 \mathrm{eV}\right)[60]$, the temperature dependence of the Ni self-diffusion coefficient was calculated. The result is presented in Fig. 5 (red solid line), where it is also compared with experimental self-diffusion coefficients. As can be seen in the figure, calculations compare well with the experimental self-diffusion coefficients at high temperatures, but deviate at lower temperatures. In Table $\mathrm{V}$, the parameters used to obtain the results of Fig. 5 are presented, as well as the resulting activation energies and prefactors. In the table, a considerable mismatch of both activation energy and prefactor can be seen, indicating that the apparent match at high temperatures might be only coincidental. Indeed, the current model is expected to perform better in the lower temperature range, as DFT results are generally more accurate in this region, and the correction factor, Eq. (17), is smaller there.

Calculations were also performed using the full set of DFT parameters obtained by Tucker et al. $\left(E_{V}^{f}=1.65 \mathrm{eV}\right.$, $E_{V}^{\text {mig }}=1.09 \mathrm{eV}, v_{0}=4.48 \mathrm{THz}$ ). The results, represented by the green line in Fig. 5, give a closer match in activation energy, but a more important deviation in prefactor, and thus no significant improvement with respect to the experimental benchmark overall.

In the above discussion, a constant vacancy formation free energy was assumed, equal to the value computed at $0 \mathrm{~K}$. However, studies by Gong et al. and Glensk et al. showed that 
finite-temperature effects can be important and may lead to strongly non-Arrhenius vacancy concentrations [61,62]. For this reason, an attempt was here made to improve the calculated self-diffusion coefficient by applying Gong's correction. Using the $0-\mathrm{K} \omega_{0}$ computed in this work, together with the vacancy concentration computed by Gong et al., which accounts for vibrational, electronic, and magnetic finite-temperature contributions to $E_{V}^{f}$, the dashed red curve in Fig. 5 was obtained.

The correction with Gong's model improves slightly the match with the experimental diffusion coefficients, both in terms of activation energy and prefactor (Table V); however, a non-negligible deviation remains, especially in the lowertemperature range. The reasons for this mismatch are hard to pinpoint. The finite-temperature effects on the vacancy migration rate $\omega_{0}$, neglected in this work, are likely to play a role; however, it is also possible that Gong's model, providing a satisfactory match at temperatures close to the melting point $(1700 \mathrm{~K})$, does not perform as well at lower temperatures.

It should be noted that calculations in this work are performed with the generalized gradient approximation (GGA). In a study by Hargather et al., the self-diffusion coefficients of fcc Ni was calculated as functions of temperature using the local density approximation (LDA) functional [69]. In their work, the authors are able to obtain a very good match with experimentally determined self-diffusion coefficients. The LDA has been previously shown to predict more accurately the activation energies for diffusion ([69] and references therein), for which reason this functional has been sometimes used to calculate such parameters. However, as vibrational entropies calculated with the GGA functional are generally considered more reliable [70], this functional is thought to give a more accurate diffusion prefactor. The choice of exchange-correlation functional when calculating diffusion coefficients is for this reason not straightforward. In the current study however, it is considered likely that the mismatch between the theoretical and experimental values is in fact due to an inaccurate description of the vacancy concentration. As indicated by Eq. (15), the quantity is based on the vacancy formation enthalpy, Eq. (10), which in turn depends in the chemical potential of the system. Thus, $E_{V}^{\mathrm{mig}}$ is a less direct comparison of DFT simulation cells compared to both migration barriers and binding energies. As a consequence of this, there is usually a stronger consensus for $E_{V}^{\mathrm{mig}}$ in the literature, and a recurring debate about the value of $E_{V}^{f}$ [60-62]. The fact that the diffusion coefficients are proportional to $c_{V}^{\mathrm{eq}}$ [Eq. (4)] further underlines the importance of a correct description of this parameter. Based on this discussion, the assumption was here made that the source of error in the calculations of Fig. 5 could be completely ascribed to an incorrect description of the vacancy concentration. $c_{V}^{\mathrm{eq}}$ was for this reason treated as a fitting parameter, which was obtained from the vacancy concentration that fits the DFT-computed values to the experimental diffusion coefficient according to Eq. (18).

$$
D_{\exp }=a_{0}^{2} f_{0} c_{V}^{\mathrm{fit}} \omega_{0} .
$$

The fitted vacancy concentration $c_{V}^{\text {fit }}$ together with the vacancy concentration obtained by the corrections according to the model proposed by Gong et al. are presented in the
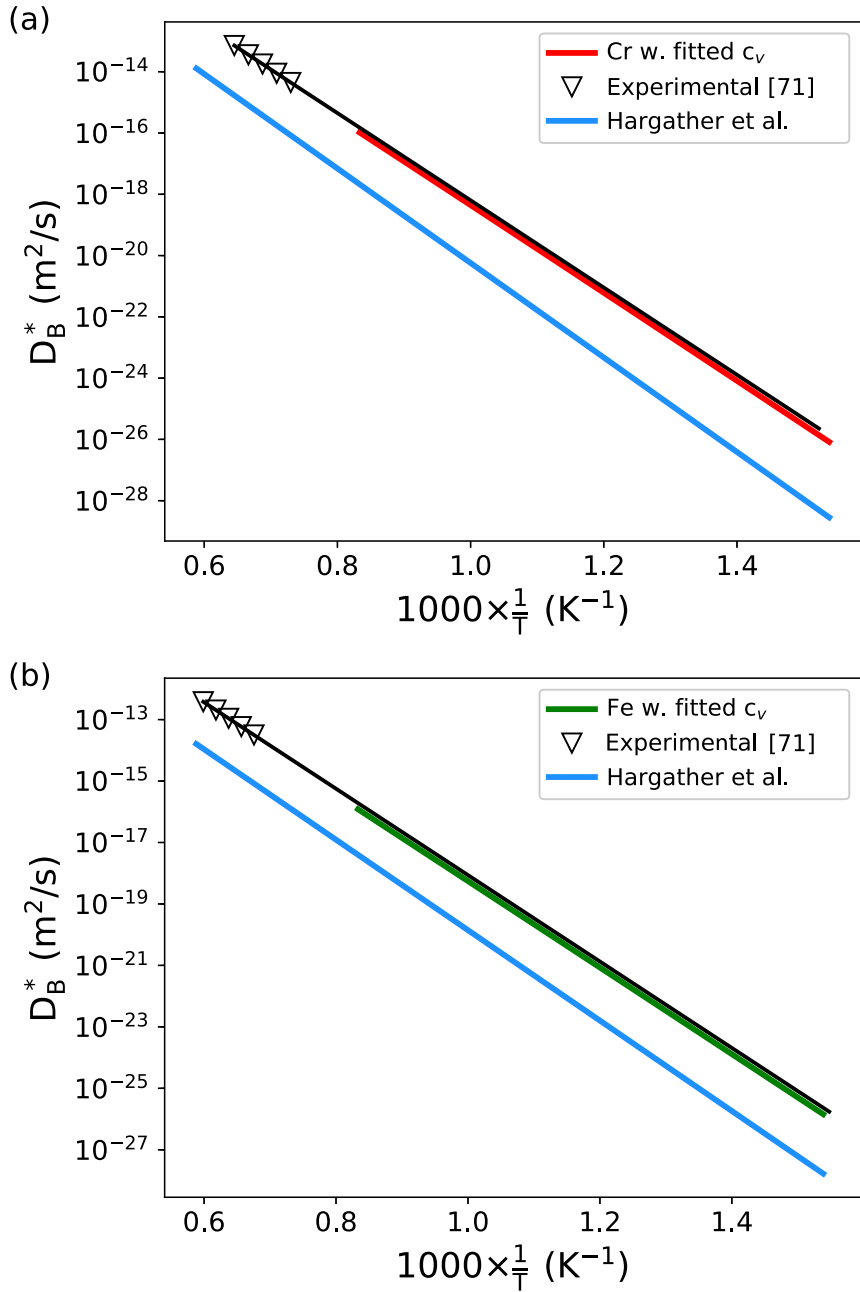

FIG. 6. Vacancy mediated tracer solute diffusion coefficient as a function of temperature for (a) $\mathrm{Cr}$ and (b) Fe. Results are compared to experimental data from [71], and with Arrhenius plot obtained with values from Hargather et al. [70]; "fitted $c_{V}$ " has been inferred from the fitting of self-diffusion coefficients shown in Fig. 5.

lower inset of Fig. 5. To verify the accuracy of the assumption that the error in Fig. 5 can be incorporated in $c_{V}$, the fitted quantity was used to calculate the solute diffusion coefficients of $\mathrm{Cr}$ and $\mathrm{Fe}$ in dilute $\mathrm{Ni}$, based on the transport coefficients obtained in this work. The results are presented in Fig. 6, together with an Arrhenius fit of experimental values from Refs. [63-68]. An extensive study on solute diffusion in dilute $\mathrm{Ni}$ has been previously performed using the five-frequency model by Hargather et al. [70]. As the authors obtained a very good fit for their calculated Ni self-diffusion coefficient in a previous work [69], their results regarding solute diffusion of $\mathrm{Cr}$ and $\mathrm{Fe}$ in $\mathrm{Ni}$ are included in the figure for reference. The figure displays a very good match between the results of this work and the experimental values. The current model also significantly improves the estimated solute diffusion coefficients for the two species compared to the Hargather study. These results indicate that the mismatch in Fig. 5 is likely due to an inaccurate estimation of $c_{V}$ at lower temperatures. For this reason, the solute-related coefficients calculated in this work are considered reliable. In addition, under irradiation, 


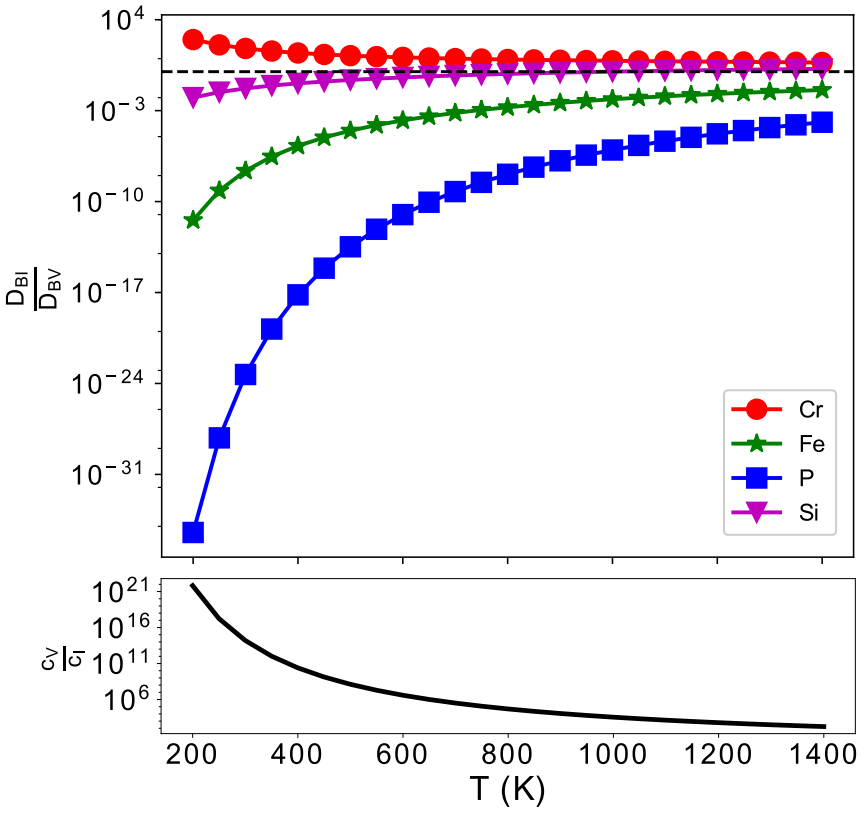

FIG. 7. Ratios of solute tracer diffusion coefficients due to vacancy and interstitial mechanism. As that interstitial diffusion is considered negligible for $\mathrm{Ti}$ and $\mathrm{Mn}$, the species are not included in the figure. The lower inset displays the ratio of vacancy and interstitial concentrations used to calculate the diffusion coefficient ratios.

radiation-induced defect formation dominates over the equilibrium defect population, so the role of $c_{V}^{\mathrm{eq}}$ in solute RIS is negligible. Hence, it can be concluded that while the calculation of vacancy equilibrium concentrations in pure $\mathrm{Ni}$ is a source of uncertainties, the solute-related properties computed in this work are reliable.

\section{Dominant diffusion mechanisms}

The dominant diffusion mechanism (vacancy or dumbbell) under thermal conditions can be determined from the ratios of the respective solute tracer diffusion coefficients, $D_{B I} / D_{B V}$. As shown in Eq. (5), the solute diffusion coefficients are proportional to the equilibrium defect concentration. The ratio $c_{V} / c_{I}$ can to a first approximation be estimated by $D_{I} / D_{V}$ [72], which at low solute concentrations reduces to $L_{I I} / L_{V V}$. Using this factor to estimate the ratio of defect concentrations, the preferred defect migration paths for all considered species migrating by both vacancy and interstitial mechanisms were assessed, and results are presented in Fig. 7. In this figure, interstitial P diffusion is based on the octahedral mechanism, which in this case is strongly coupled with the diffusion of pure Ni dumbbells, as the latter are required to kick out $\mathrm{P}$ into the octahedral configuration. For all other species, the interstitial migration is solely based on the dumbbell mechanism.

As can be seen in Fig. 7, vacancy-mediated migration is dominant for all species but $\mathrm{Cr}$, where the dumbbell mechanism is more important. By assuming a constant vacancy-to-solvent fraction of $10^{-6}$, to simulate irradiation conditions, the solute diffusion coefficients of the preferred mechanisms were computed. As $\mathrm{Cr}$ shows a preference to interstitial migration, the interstitial concentration was assessed

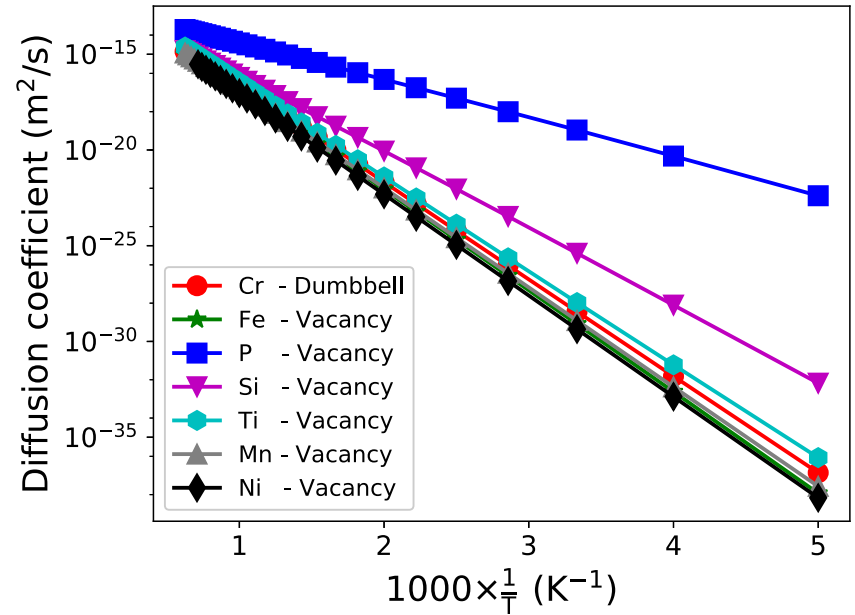

FIG. 8. Diffusion coefficients by the preferred diffusion mechanism for the various species. Calculations were performed while assuming a constant vacancy to solvent fraction of $10^{-6}$.

from the same vacancy ratio, followed by the assumption that $c_{V} / c_{I}=L_{I I} / L_{V V}$ [72]. Results are presented in Fig. 8. The figure shows that $\mathrm{P}$ is by far the fastest diffuser. Interestingly, $\mathrm{P}$ has also been shown to be faster than both $\mathrm{Cr}, \mathrm{Mn}, \mathrm{Ni}$, and $\mathrm{Si}$ in bcc Fe [34]. Although in the latter case, the migration of $\mathrm{P}$ is dominated by the dumbbell mechanism, fast migration occurs only by vacancies. In Fig. 8 it can be seen that $\mathrm{Si}$ is faster than the other solutes with the exception of P. All other solutes have similar diffusion rates to one another, which are also close to that of $\mathrm{Ni}$ self-diffusion.

\section{Partial diffusion coefficients and radiation-induced segregation}

In Fig. 9 the conventional vacancy drag ratios, $G_{V}=$ $L_{V B}^{(V B)} / L_{B B}^{(V B)}$, are presented together with the partial diffusion coefficients (PDCs) for vacancy and dumbbell mediated diffusion. The drag ratio indicates if a solute is likely to follow the vacancy in its migration, $G_{V}>1$, or if it will diffuse in the opposite direction, $G_{V}<1$. The PDCs show the enrichment/depletion tendency of a solute, induced by one diffusion mechanism while assuming that the other mechanism is inactive. In the case of vacancy-coupled diffusion, enrichment of a species can occur even in the absence of drag, as a result of competition with the surrounding bulk atoms. This is indicated by $0 \leqslant \mathrm{PDC}_{\mathrm{vac}} \leqslant 1$. See Sec. II B for a full description of the PDCs.

$\mathrm{P}$ and $\mathrm{Si}$ are shown in Fig. 9 to enrich at sinks by vacancy drag at all temperatures considered in this study. This result is expected based on observations in Secs. III A and III B, where positive solute-vacancy binding, and a higher probability of vacancy-solute pair association than dissociation, were found for the two species. The combination of the two factors leads to diffusion of the vacancy-solute pairs as coupled species, thus explaining the vacancy-mediated enrichment. In the case of Ti, results in Fig. 9 show a switchover at $320 \mathrm{~K}$ between enrichment due to vacancy drag, and depletion in the absence of drag. Table II indicates a weak $1 \mathrm{nn}$ binding interaction between $\mathrm{Ti}$ and vacancies, and in Table III it is shown that the Ti-vacancy migration barrier $\omega_{2}$ is approximately $50 \%$ as high as the $\omega_{1,3-5}$ barriers. As discussed in Sec. III B, 

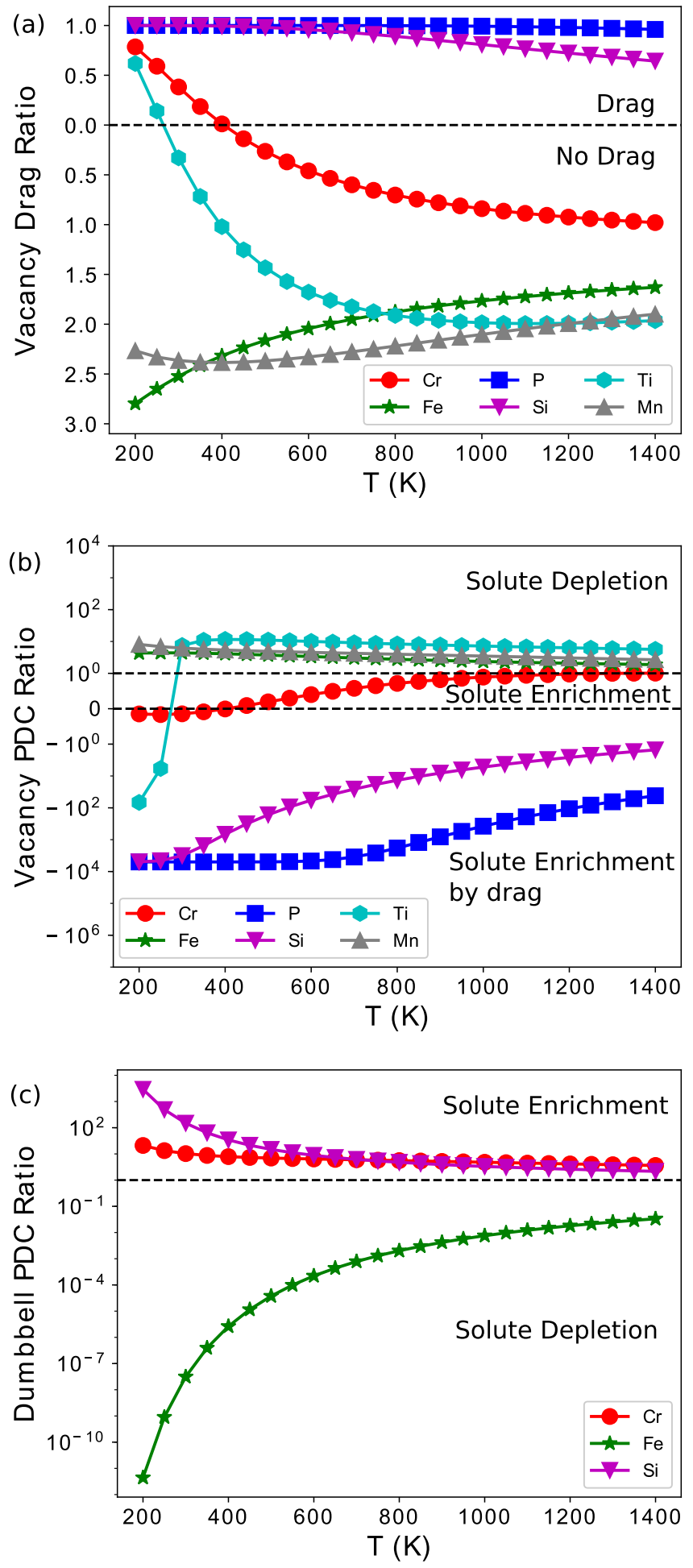

FIG. 9. (a) Vacancy drag ratio, $G_{V}=L_{V B}^{(V B)} / L_{B B}^{(V B)}$, (b) vacancy partial diffusion coefficient ratios, and (c) dumbbell partial diffusion coefficient ratios as functions of temperature. As interstitial diffusion is considered negligible for $\mathrm{Ti}$ and $\mathrm{Mn}$, and the octahedral kickout mechanism dominates over the dumbbell mechanism for $\mathrm{P}$, the three are omitted in (c).

if $\omega_{2}$ is considerably lower than the other barriers, preferential solute-vacancy exchange can prevent net transport in the material. This behavior is incorporated in the correlation factor, $f_{B}$ of Eq. (5), whose temperature dependence follows an Arrhenius behavior and can, for this reason, be seen as an additional contribution to the migration activation energy. For $\mathrm{Ti}$, this contribution was calculated to $E_{f}=0.52 \mathrm{eV}$, which in combination with the original $\omega_{2}$ results in an effective barrier of $1.02 \mathrm{eV}$. This can be expected to slow down diffusion, but as the vacancy diffusion rate is affected to an equal extent as that of the solute, the effective barrier does not explain the crossover in drag/no drag demonstrated in Fig. 9. This behavior is instead likely due to the combination of a weak Ti-1 nn binding and Ti-2 nn repulsion. Indeed, a similar switchover has been observed for vacancy mediated diffusion of Ti in bcc $\mathrm{Fe}$, where a weak $1 \mathrm{nn}$ binding and a $2 \mathrm{nn}$ repulsion have also been demonstrated [55].

In Fig. 9, it can be seen that both $\mathrm{Fe}$ and $\mathrm{Mn}$ are depleted due to the inverse-Kirkendall mechanism, in which vacancies and solutes move in opposite directions. The opposite behavior is observed in the lower temperature range for $\mathrm{Cr}$, which is dragged by vacancies up to a crossover temperature of 400 K. However, as indicated by Fig. 9(b), $\mathrm{Cr}$ is enriched up to temperatures of approximately $1000 \mathrm{~K}$ as a consequence of $\mathrm{Ni}$ being more effective at diffusing away from the sinks. The above results are in line with previous results by Tucker $e t$ al., where a crossover temperature of approximately $460 \mathrm{~K}$ was obtained for $\mathrm{Cr}$ drag by vacancies, and the inverse-Kirkendall mechanism was found for $\mathrm{Fe}$ at all temperatures [60].

In the case of dumbbell mediated diffusion, Fig. 9(c) displays enrichment of $\mathrm{Cr}$ and $\mathrm{Si}$, and depletion of $\mathrm{Fe}$. As can be seen in Table II, both $\mathrm{Si}$ and $\mathrm{Cr}$ form stable mixed $\langle 100\rangle$ dumbbells. Table IV also shows that $\mathrm{Cr}$ and $\mathrm{Si}$ generally have lower association energy barriers compared to those of dissociation. Fe, on the other hand repels the dumbbell configuration, whereas the various association/dissociation barriers are similar for this species. Thus results seen in Fig. 9(c) are in line with what could have been expected based on the DFT study in the first part of this work. It should be noted that the $\mathrm{Fe} \mathrm{PDC}_{\text {dumb }}$ is very small compared to those of $\mathrm{Si}$ and Cr. This result supports the discussion of Sec. III A, according to which repulsive interactions of the mixed $\mathrm{Ti}$ and $\mathrm{Mn}\langle 100\rangle$ dumbbells were seen as indication of negligible SIA diffusion for the two species. Since the SIA-Ti and the SIA-Mn interactions are even more negative compared to $\mathrm{Fe}$, the two will confidently not be subjected to interstitial diffusion.

Once the segregation tendencies of vacancy- and interstitial mediated diffusion have been determined through the PDCs, the overall RIS behavior can be estimated. RIS tendencies are described by $\alpha$, defined in Eq. (3). If the factor is positive, enrichment of the solute occurs at defect sinks, whereas a negative factor describes depletion. The RIS factors of the solutes considered in this work are presented in Fig. 10 as functions of temperature. $\mathrm{Ti}$ and $\mathrm{Mn}$ were assumed not to have any transport by interstitials, which was accounted for by setting the corresponding $L_{I B}$ and $L_{B B}$ of Eq. (3) to zero, and $L_{I I}$ is that of pure Ni dumbbell migration. This makes the RIS factors for the two species independent of interstitial migration. In the case of $\mathrm{P}$ interstitial migration, only the octahedral configuration was considered. As discussed in Sec. II B, this configuration forms spontaneously if a migrating Ni dumbbell encounters a substitutional $\mathrm{P}$ in the lattice, as one of the $\mathrm{Ni}$ atoms takes the original place of the $\mathrm{P}$ atom. 


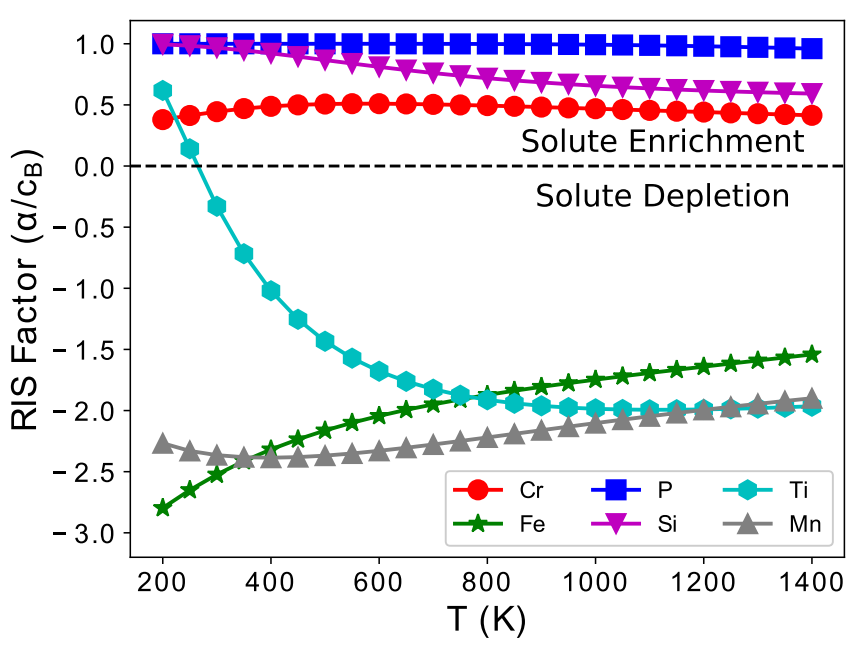

FIG. 10. RIS tendencies of $\mathrm{Cr}, \mathrm{Fe}, \mathrm{P}, \mathrm{Si}, \mathrm{Ti}$, and $\mathrm{Mn}$ in bulk nickel. Positive values indicate enrichment at sinks.

\section{DISCUSSION}

Previous studies of austenitic alloys show Ni, Si, and $\mathrm{P}$ enrichment, and $\mathrm{Fe}, \mathrm{Cr}$, Mn depletion at grain boundaries and interfaces due to RIS [73-81]. In Ni alloys, Cr enrichment and Fe depletion have been detected at sinks [79,82,83], which is in line with the results of this work. As shown in Fig. 10, $\mathrm{Fe}$ and $\mathrm{Mn}$ are the only species which are depleted at all temperatures, since both diffuse via the inverse-Kirkendall mechanism. Interstitial diffusion is shown in Fig. 9 to give rise to Fe depletion, resulting from the influx of pure Ni dumbbells towards sinks. Based on the magnitudes of the PDC ratios in Fig. 9, it can however be assumed that the interstitial contribution is less important than that of the vacancy mechanism for the overall RIS of Fe in fcc Ni [Eq. (3)]. As the results in Fig. 7 show that the vacancy mechanism is also dominant in the absence of radiation, it can be concluded that in the case of $\mathrm{Fe}$ migration in $\mathrm{Ni}$, the vacancy mechanism is dominant both in thermal and nonequilibrium conditions. Results regarding $\mathrm{Fe}$ in this study are not directly applicable to fcc Fe alloys, as the behavior of $\mathrm{Fe}$ in $\mathrm{Ni}$ can be very different from the behavior of $\mathrm{Ni}$ in Fe. However, in the case of Ni precipitation in austenitic steels, Fe atoms inside such a precipitate will see a Ni-rich environment. In this case, Fe is likely to behave as predicted in this study, i.e., it will deplete from the precipitate.

In the case of $\mathrm{Si}$, results in this work show that both interstitial and vacancy mechanisms contribute to its enrichment. Enrichment at sinks has been observed experimentally following irradiation of $\mathrm{Ni}$ and fcc Fe alloys [18,19,74,75,84,85]. From the PDC ratios of this work, one can conclude that the vacancy mechanism dominates the observed behavior up to temperatures of $\sim 1200 \mathrm{~K}$, where the interstitial mechanism becomes slightly more important. A similar behavior is observed in thermal conditions, as Fig. 7 displays a slight preference for the vacancy mechanism, although the difference is negligible at higher temperatures. One can conclude that the experimental observations are likely due to the vacancy mechanism. As the same mechanism dominates Si migration in irradiated bcc Fe [34], one can suspect a pronounced vacancy contribution to the observed $\mathrm{Si}$ enrichment in fcc Fe alloys.

$\mathrm{P}$ displays enrichment due to both vacancy and octahedral mechanisms. From results in Fig. 7, and the magnitudes of the $\mathrm{PDC}$ ratios in Fig. 9, it can be concluded that $\mathrm{P}$ diffusion is dominated by the vacancy mechanism. This is consistent with the significant migration barrier, $0.95 \mathrm{eV}$, for octahedral diffusion. Segregation of $\mathrm{P}$ has also been observed in bcc Fe, and is a well-known problem in austenitic steels. The preferred migration mechanism of $\mathrm{P}$ is different in fcc Ni compared to bcc Fe, where enrichment of $\mathrm{P}$ is dominated by the dumbbell mechanism [34]. However, $\mathrm{P}$ displays strong tendencies for vacancy drag in both materials. As the mixed Ni-P dumbbell is not stable in fcc $\mathrm{Ni}$, one can suspect that the vacancy mechanism is more important in austenitic steel compared to bcc $F e$. However, the stability of the various mixed $\mathrm{P}-X$ dumbbells in austenitic alloys remains to be verified in order to safely determine the relative importance of the two mechanisms in the material.

Based on DFT results in Sec. III A, it can be concluded that $\mathrm{Ti}$ does not migrate via the interstitial mechanism, and that only the vacancy mechanism contributes to RIS for this species. In bcc Fe, Ti diffusion by dumbbells has been shown to be negligible due to repulsion of the Fe-Ti dumbbell [55]. Additionally, the crossover for enrichment/depletion at $320 \mathrm{~K}$ displayed in Fig. 10 resembles observations of $\mathrm{Ti}$ in bcc Fe, where a switchover between enrichment and depletion has been found at approximately $700 \mathrm{~K}$ [55]. Thus, the behavior of $\mathrm{Ti}$ in fcc $\mathrm{Ni}$ and in bcc Fe bears significant similarities. However, the crossover temperature of $320 \mathrm{~K}$ in $\mathrm{Ni}$ is well below reactor operating temperatures, whereas the crossover temperature in $\mathrm{Fe}$ is not. This gives rise to difficulties in transposing observations to austenitic alloys used in current NPPs. Nevertheless, Ti has previously been shown to prevent swelling of austenitic materials in reactor applications [86-89] and to have a stabilizing effect on voids in Ni-based model alloys [90]. Results in this work indicate that a possible explanation for these observations is the trapping of vacancies due to the preferential exchange with Ti. A more thorough investigation of vacancy trapping by Ti would entail the computation of the vacancy diffusion coefficient as a function of Ti concentration, but this is beyond the scope of this work.

In Fig. 10, $\mathrm{Cr}$ is shown to enrich at sinks as a consequence of both to the interstitial and vacancy mechanisms. Based on diffusion coefficients and PDC ratios, it can be concluded that $\mathrm{Cr}$ preferentially diffuses by the dumbbell migration. Previous studies of $\mathrm{Cr}$ in fcc $\mathrm{Ni}$ generally detect depletion at sinks, however enrichment has been observed in a work by Allen et al. [77]. In their study, RIS in three different Ni based alloys (Ni-18Cr, Ni-18Cr-9Fe, and Ni-18Cr-0.08P) was examined by exposing the materials to either thermal treatment or proton irradiation. $\mathrm{Cr}$ depletion was observed for all samples, with the exception of one, where enrichment at sinks was observed. The authors of the study suggested that the behavior of $\mathrm{Cr}$ is very sensitive to interactions with the surroundings. In bcc Fe, the dumbbell mechanism results in enrichment and vacancy mechanism in depletion of $\mathrm{Cr}$ near sinks [34,91]. However, the dominant mechanism and overall evolution of $\mathrm{Cr}$ were again reported to be very sensitive to temperature, sink density, $\mathrm{Cr}$ concentration, and local strain fields [34,91,92]. The 
sensitivity of $\mathrm{Cr}$ to external conditions, both in fcc $\mathrm{Ni}$ and bcc $\mathrm{Fe}$, gives rise to difficulties in transposing this work to fcc $\mathrm{Fe}$ and its alloys.

Results here presented indicate that vacancy-mediated diffusion is dominant for all species in fcc $\mathrm{Ni}$, with the exception of $\mathrm{Cr}$ where the dumbbell mechanism is prominent. The dominant diffusion mechanism does not change for either species depending on whether thermal or nonequilibrium conditions are considered. Interestingly, the vacancy dominated migration in $\mathrm{Ni}$ is in line with the common explanation that RIS in austenitic Fe alloys occurs as a consequence of the inverse Kirkendall mechanism [93-95].

Characterizations of irradiated austenitic alloys following reactor service show a high number density of $\mathrm{Ni}$-Si enriched and $\mathrm{Cr}-\mathrm{Fe}$ depleted clusters, together with $\mathrm{P}$ segregation at the interfaces of the clusters [74] or RIS-induced Ni enrichment in the vicinity of grain boundaries. Although not directly applicable to austenitic alloys, results in the current study are relevant for transport phenomena occurring inside and near the internal surface of such clusters or in the vicinity of segregated grain boundaries. A natural continuation of this work would be to perform a similar study in fcc Fe, as this would give a more precise insight on the impact of the lattice structure on segregation tendencies. In future works, the effect of local composition could also be included in order to evaluate the segregation tendencies as functions of solute concentration. With that said, this study provides valuable information for improving the current understanding of RIS in Ni-based alloys.

\section{CONCLUSION}

Solute diffusion, flux coupling, and radiation-induced segregation (RIS) of $\mathrm{Fe}, \mathrm{Cr}, \mathrm{P}, \mathrm{Si}, \mathrm{Ti}$, and $\mathrm{Mn}$ in fcc $\mathrm{Ni}$ have here been investigated by coupling first-principles calculations with the self-consistent mean field theory. The goal has been to improve the current understanding of radiationinduced segregation processes of materials commonly used in today's and future generation nuclear power plants. For this reason, findings have been compared with similar studies in bcc Fe, and discussed in the context of RIS in austenitic steel. Results show that interstitial migration has little impact on solute diffusion in fcc $\mathrm{Ni}$ compared to that of vacancies, with the exception of $\mathrm{Cr}$, for which the migration is dominated by a dumbbell mechanism. In addition, it has here been shown that $\mathrm{P}, \mathrm{Si}$, and $\mathrm{Cr}$ are enriched at sinks as a consequence of radiation-induced segregation, whereas $\mathrm{Fe}$ and $\mathrm{Mn}$ are depleted. Ti was shown to enrich at sinks at low temperatures, with a switchover near room temperature, followed by depletion in the higher temperature range. Results in fcc Ni are to a great extent in line with observations in bcc Fe, where Si and Ti migration are dominated by the vacancy mechanism, and $\mathrm{Cr}$ dumbbell migration leads to enrichment at sinks. Interstitial $\mathrm{P}$ however, behaves very differently in fcc Ni compared to bcc Fe. In Fe, the solute forms a quickly migrating, hardly dissociating mixed dumbbell, whereas in $\mathrm{Ni}$, substitutional $\mathrm{P}$ will preferentially be kicked out into a more slowly migrating octahedral configuration.

This work has improved the understanding of the underlying atomic-transport phenomena behind solute segregation in irradiated materials. As calculations were performed in the dilute limit, the results are not directly applicable to concentrated Ni alloys in current nuclear power plants. However, this comprehensive modeling-based analysis demonstrates RIS tendencies due to both vacancy and dumbbell mechanisms in $\mathrm{Ni}$ alloys. The results shed a light on the intrinsic kinetic behavior of several important solutes in Ni alloys and austenitic steels, and can for this reason be considered an important milestone towards a broader picture of irradiation damage in nuclear structural materials.

\section{ACKNOWLEDGMENTS}

This work was partly financed by the Euratom research and training program 2014-2018 under Grant Agreements No. 755269 and No. 633053 as well as the Swedish Centre for Nuclear Technology (SKC). We acknowledge EDF R\&D HPC resources as well as the CINECA award under the ISCRA initiative, for the availability of high performance computing resources and support. The computations were also enabled by resources provided by the Swedish National Infrastructure for Computing (SNIC) at KTH Royal Institute of Technology partially funded by the Swedish Research Council through Grant Agreement No. 2016-07213. We also acknowledge PRACE for awarding us access to resource MARCONI-KNL at CINECA in Italy and Hazel Hen at HLRS in Germany [project MORPHO (modeling radiation damage: characterization of elementary physical processes), Grant No. 2016153636].
[1] D. Lemarchand, E. Cadel, S. Chambreland, and D. Blavette, Philos. Mag. A 82, 1651 (2002).

[2] A. F. Rowcliffe, L. K. Mansur, D. T. Hoelzer, and R. K. Nanstad, J. Nucl. Mater. 392, 341 (2009).

[3] K. Rudy and R. E. Stoller, Comprehensive Nuclear Materials, edited by R. J. M. Konings (Elsevier, Oxford, 2020).

[4] G. S. Was, Fundamentals of Radiation Materials Science: Metals and Alloys (Springer, New York, 2016).

[5] Commissariat à l'énergie atomique et aux énergies alternatives (CEA), Nuclear Materials Structural Materials Modeling and Simulation, edited by Jean-François
Parisot (CEA Paris-Saclay and Groupe Moniteur, Paris, 2020).

[6] J. L. Brimhall, D. R. Baer, and R. H. Jones, J. Nucl. Mater. 117, 218 (1983).

[7] K. Jin, W. Guo, C. Lu, M. W. Ullah, Y. Zhang, W. J. Weber, L. Wang, J. D. Poplawsky, and H. Bei, Acta Mater. 121, 365 (2016).

[8] M. W. Ullah, D. S. Aidhy, Y. Zhang, and W. J. Weber, Acta Mater. 109, 17 (2016).

[9] D. Chakraborty and D. S. Aidhy, J. Alloys Compd. 725, 449 (2017). 
[10] Y. Yang, K. G. Field, T. R. Allen, and J. T. Busby, J. Nucl. Mater. 473, 35 (2016).

[11] E. Levo, F. Granberg, C. Fridlund, K. Nordlund, and F. Djurabekova, J. Nucl. Mater. 490, 323 (2017).

[12] T. Yang, C. Lu, G. Velisa, K. Jin, P. Xiu, M. L. Crespillo, Y. Zhang, H. Bei, and L. Wang, Acta Mater. 151, 159 (2018).

[13] C. Lu, K. Jin, L. K. Béland, F. Zhang, T. Yang, L. Qiao, Y. Zhang, H. Bei, H. M. Christen, R. E. Stoller, and L. Wang, Sci. Rep. 6, 19994 (2016).

[14] K. Sato, D. Itoh, T. Yoshiie, Q. Xu, A. Taniguchi, and T. Toyama, J. Nucl. Mater. 417, 963 (2011).

[15] T. Yoshiie, Q. Xu, Y. Satoh, H. Ohkubo, and M. Kiritani, J. Nucl. Mater. 283, 229 (2000).

[16] T. Yoshiie, T. Ishizaki, Q. Xu, Y. Satoh, and M. Kiritani, J. Nucl. Mater. 307, 924 (2002).

[17] M. Kiritani, T. Yoshiie, M. Iseki, S. Kojima, K. Hamada, M. Horiki, Y. Kizuka, H. Inoue, T. Tada, and Y. Ogasawara, J. Nucl. Mater. 212, 241 (1994).

[18] L. E. Rehn, P. R. Okamoto, and H. Wiedersich, J. Nucl. Mater. 80, 172 (1979).

[19] R. S. Averback, L. E. Rehn, W. Wagner, H. Wiedersich, and P. R. Okamoto, Phys. Rev. B 28, 3100 (1983).

[20] M. Yamaguchi, M. Shiga, and H. Kaburaki, J. Phys.: Condens. Matter 16, 3933 (2004).

[21] M. V̌̌ianská and M. Šob, Prog. Mater. Sci. 56, 817 (2011).

[22] S. G. Druce, G. Gage, and G. Jordan, Acta Metall. 34, 641 (1986).

[23] W. T. Geng, A. J. Freeman, R. Wu, C. B. Geller, and J. E. Raynolds, Phys. Rev. B 60, 7149 (1999).

[24] J. Dong, M. Zhang, X. Xie, and R. G. Thompson, Mater. Sci. Eng. A 328, 8 (2002).

[25] L. E. Rehn, P. R. Okamoto, D. I. Potter, and H. Wiedersich, J. Nucl. Mater. 74, 242 (1978).

[26] E. T. Bentria, I. K. Lefkaier, A. Benghia, B. Bentria, M. B. Kanoun, and S. Goumri-Said, Sci. Rep. 9, 14024 (2019).

[27] Effects of Radiation on Materials: 14th International Symposium, edited by N. Packan, R. Stoller, and A. Kumar (ASTM International, West Conshohocken, PA, 1990), Vol. I.

[28] A. R. Allnatt and A. B. Lidiard, in Atomic Transport in Solids (Cambridge University Press, Cambridge, UK, 2003), pp. 1518.

[29] M. Nastar, Philos. Mag. 85, 3767 (2005).

[30] T. Garnier, D. R. Trinkle, M. Nastar, and P. Bellon, Phys. Rev. B 89, 144202 (2014).

[31] D. R. Trinkle, Philos. Mag. 97, 2514 (2017).

[32] V. Barbe and M. Nastar, Philos. Mag. 86, 1513 (2006).

[33] T. Schuler, L. Messina, and M. Nastar, Comput. Mater. Sci. 172, 109191 (2020).

[34] L. Messina, T. Schuler, M. Nastar, M.-C. Marinica, and P. Olsson, Acta Mater. 191, 166 (2020).

[35] A. D. Le Claire, J. Nucl. Mater. 69, 70 (1978).

[36] M. Nastar and F. Soisson, in Comprehensive Nuclear Materials, edited by R. J. M. Konings (Elsevier, Oxford, 2012), pp. 471496.

[37] E. Martínez, O. Senninger, A. Caro, F. Soisson, M. Nastar, and B. P. Uberuaga, Phys. Rev. Lett. 120, 106101 (2018).

[38] T. Schuler and M. Nastar, Phys. Rev. B 93, 224101 (2016).

[39] A. Van der Ven and G. Ceder, Phys. Rev. Lett. 94, 045901 (2005).

[40] G. Kresse and J. Hafner, Phys. Rev. B 47, 558 (1993).
[41] G. Kresse and J. Furthmüller, Phys. Rev. B 54, 11169 (1996).

[42] J. P. Perdew, K. Burke, and M. Ernzerhof, Phys. Rev. Lett. 77, 3865 (1996).

[43] G. Mills, H. Jónsson, and G. K. Schenter, Surf. Sci. 324, 305 (1995).

[44] G. H. Vineyard, J. Phys. Chem. Solids 3, 121 (1957).

[45] G. Henkelman, B. P. Uberuaga, and H. Jónsson, J. Chem. Phys. 113, 9901 (2000).

[46] A. Togo and I. Tanaka, Scr. Mater. 108, 1 (2015).

[47] R. Nazarov, T. Hickel, and J. Neugebauer, Phys. Rev. B 85 , 144118 (2012).

[48] R. A. Johnson and A. N. Orlov, Physics of Radiation Effects in Crystals (Elsevier, New York, 2012).

[49] C.-C. Fu, F. Willaime, and P. Ordejón, Phys. Rev. Lett. 92, 175503 (2004).

[50] T. P. C. Klaver, D. J. Hepburn, and G. J. Ackland, Phys. Rev. B 85, 174111 (2012).

[51] J. B. Piochaud, T. P. C. Klaver, G. Adjanor, P. Olsson, C. Domain, and C. S. Becquart, Phys. Rev. B 89, 024101 (2014).

[52] C. Domain and C. S. Becquart, Phys. Rev. B 71, 214109 (2005).

[53] F. Maury, P. Lucasson, A. Lucasson, F. Faudot, and J. Bigot, J. Phys. F: Met. Phys. 17, 1143 (1987).

[54] E. Meslin, C.-C. Fu, A. Barbu, F. Gao, and F. Willaime, Phys. Rev. B 75, 094303 (2007).

[55] L. Messina, M. Nastar, N. Sandberg, and P. Olsson, Phys. Rev. B 93, 184302 (2016).

[56] H. Wu, T. Mayeshiba, and D. Morgan, Sci. Data 3, 160054 (2016).

[57] P. Olsson, T. P. C. Klaver, and C. Domain, Phys. Rev. B 81, 054102 (2010).

[58] L. Messina, M. Nastar, T. Garnier, C. Domain, and P. Olsson, Phys. Rev. B 90, 104203 (2014).

[59] M.-C. Marinica, C. Barreteau, D. Spanjaard, and M.-C. Desjonquères, Phys. Rev. B 72, 115402 (2005).

[60] J. D. Tucker, R. Najafabadi, T. R. Allen, and D. Morgan, J. Nucl. Mater. 405, 216 (2010).

[61] Y. Gong, B. Grabowski, A. Glensk, F. Körmann, J. Neugebauer, and R. C. Reed, Phys. Rev. B 97, 214106 (2018).

[62] A. Glensk, B. Grabowski, T. Hickel, and J. Neugebauer, Phys. Rev. X 4, 011018, (2014).

[63] A. R. Wazzan and J. E. Dorn, J. Appl. Phys. 36, 3596 (1965).

[64] I. G. Ivantsov, Phys. Met. Metallogr. USSR 5, 77 (1966) [Fiz. Metal. Metalloved. 22, 725 (1966)].

[65] H. Bakker, Phys. Status Solidi B 28, 569 (1968).

[66] M. Feller-Kniepmeier, M. Gründler, and H. Helfmeier, Z. Metallkd. 67, 533 (1976).

[67] K. Maier, H. Mehrer, E. Lessmann, and W. Schiile, Phys. Status Solidi B 78, 689 (1976).

[68] A. B. Vladimirov, V. N. Kaigorodov, S. M. Klotsman, and I. S. Tracktenberg, in DIMETA 82, Proceedings of the International Conference on Diffusion in Metals and Alloys, edited by E. J. Kedves and D. L. Beke (Trans Tech Publications, Zurich, Switzerland, 1983), p. 338.

[69] C. Z. Hargather, S.-L. Shang, Z.-K. Liu, and Y. Du, Comput. Mater. Sci. 86, 17 (2014).

[70] C. Z. Hargather, S.-L. Shang, and Z.-K. Liu, Acta Mater. 157, 126 (2018).

[71] H. Mehrer, N. Stolica, and N. A. Stolwijk, in Diffusion in Solid Metals and Alloys, Landolt-Börnstein, Group III: Condensed 
Matter, edited by H. Mehrer (Springer-Verlag, Berlin, 1990), Vol. 26, p. 68.

[72] R. Sizmann, J. Nucl. Mater. 69, 386 (1978).

[73] G. S. Was, J. P. Wharry, B. Frisbie, B. D. Wirth, D. Morgan, J. D. Tucker, and T. R. Allen, J. Nucl. Mater. 411, 41 (2011).

[74] A. Etienne, B. Radiguet, P. Pareige, J.-P. Massoud, and C. Pokor, J. Nucl. Mater. 382, 64 (2008).

[75] A. Etienne, B. Radiguet, N. J. Cunningham, G. R. Odette, and P. Pareige, J. Nucl. Mater. 406, 244 (2010).

[76] C. Lu, T. Yang, K. Jin, N. Gao, P. Xiu, Y. Zhang, F. Gao, H. Bei, W. J. Weber, K. Sun, Y. Dong, and L. Wang, Acta Mater. 127, 98 (2017).

[77] T. R. Allen, L. Tan, G. S. Was, and E. A. Kenik, J. Nucl. Mater. 361, 160 (2007).

[78] N. Sakaguchi, H. Takahashi, and H. Ichinose, Mater. Trans. 46, 440 (2005)

[79] L. Barnard, J. D. Tucker, S. Choudhury, T. R. Allen, and D. Morgan, J. Nucl. Mater. 425, 8 (2012).

[80] M. Hatakeyama, S. Tamura, and I. Yamagata, Mater. Lett. 122, 301 (2014).

[81] Z. Jiao and G. S. Was, Acta Mater. 59, 1220 (2011).

[82] X.-X. Wang, L.-L. Niu, and S. Wang, Mater. Lett. 202, 120 (2017).
[83] A. Barashev, Y. Osetsky, H. Bei, C. Lu, L. Wang, and Y. Zhang, Curr. Opin. Solid State Mater. Sci. 23, 92 (2019).

[84] T. Ezawa, E. Wakai, and R. Oshima, J. Nucl. Mater. 283, 244 (2000).

[85] P. K. Rastogi and A. J. Ardell, Acta Metall. 19, 321 (1971).

[86] D. J. Mazey, D. R. Harries, and J. A. Hudson, J. Nucl. Mater. 89, 155 (1980).

[87] A. Hishinuma and K. Fukai, J. Nucl. Sci. Technol. 20, 668 (1983).

[88] R. M. Boothby and T. M. Williams, J. Nucl. Mater. 152, 123 (1988).

[89] H. Kawanishi, M. Yamada, K. Fukuya, and S. Ishino, J. Nucl. Mater. 104, 1097 (1981).

[90] K. Ma, B. Décamps, A. Fraczkiewicz, F. Prima, and M. LoyerProst, Mater. Res. Lett. 8, 201 (2020).

[91] O. Senninger, F. Soisson, E. Martínez, M. Nastar, C.-C. Fu, and Y. Bréchet, Acta Mater. 103, 1 (2016)

[92] F. Soisson, E. Meslin, and O. Tissot, J. Nucl. Mater. 508, 583 (2018).

[93] T. R. Allen, J. T. Busby, G. S. Was, and E. A. Kenik, J. Nucl. Mater. 255, 44 (1998).

[94] T. R. Allen and G. S. Was, Acta Mater. 46, 3679 (1998).

[95] J. P. Wharry and G. S. Was, J. Nucl. Mater. 442, 7 (2013). 